\title{
Comparison Bayes Estimators of Reliability in the Exponential Distribution
}

\author{
Dr. Jinan Abbas Naser Al- obedy -Assistant professor
}

Technical College of Management-Baghdad

\author{
تاريخ التقديم:2016/9/27 \\ تاريخ القبول:2017/1/11
}

\section{Abstract}

We produced a study in Estimation for Reliability of the Exponential distribution based on the Bayesian approach. These estimates are derived using Bayesian approaches. In the Bayesian approach, the parameter of the Exponential distribution is assumed to be random variable .we derived bayes estimators of reliability under four types when the prior distribution for the scale parameter of the Exponential distribution is: Inverse Chi-square distribution, Inverted Gamma distribution, improper distribution, Non-informative distribution. And estimators for Reliability is obtained using the well known squared error loss function and weighted squared errors loss function. We used simulation technique, to compare the resultant estimators in terms of their mean squared errors (MSE), mean weighted squared errors (MWSE).Several cases assumed for the parameter of the exponential distribution for data generating, of different samples sizes (small, medium, and large). The results were obtained by using simulation technique, Programs written using MATLAB-R2008a program were used. In general, Simulation results shown that the resultant estimators in terms of their mean squared errors (MSE) is better than the resultant estimators in terms of their mean weighted squared errors (MWSE).According to the our criteria is the best estimator that gives the smallest value of MSE or MWSE . For example bayes estimation is the best when the prior distribution for the scale parameter is improper and Non-informative distributions according to the smallest value of MSE comparative to the values of MWSE for all samples sizes at some of true value of $t$ and $\theta$.

Key words: The Exponential, Bayes method, the prior distributions: Inverse Chi-square distribution, Inverted Gamma distribution, improper distribution, non-informative distribution, mean squared errors (MSE), mean weighted squared errors (MWSE).

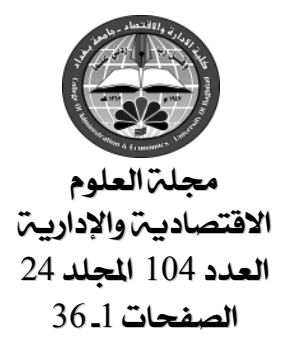




\section{Comparison Bayes Estimators of Reliability in the Exponential Distribution}

\section{Introduction}

The exponential distribution is one of the most important distributions in lifetesting and reliability studies. Inference procedures for the exponential distribution and applications in the context of life-testing and reliability have been discussed by many authors. We mention some of them in a brief manner:

Chiou (1993) ${ }^{[4]}$ propose two empirical Bayes shrinkage estimators for the reliability of the exponential distribution and study their properties. Under the uniform prior distribution and the inverted gamma prior distribution these estimators are developed and compared with a preliminary test estimator and with a shrinkage estimator in terms of mean squared error. The proposed empirical Bayes shrinkage estimator under the inverted gamma prior distribution is shown to be preferable to the preliminary test estimator and the shrinkage estimator when the prior value of mean life is close to the true mean life.

Baklizi (2003) ${ }^{[1]}$ investigated the advantages of incorporating prior information in the reliability function through the shrinkage estimators. His work is an effort to coin unified shrinkage estimators of reliability function for five lifetime distributions commonly used to model lifetime data by biologists, physicists, engineers and statisticians for living and non-living entities.

Sarhan (2003) ${ }^{[7]}$ exploits past experiments to approximate a prior information (prior density) into

the model, in estimating reliability function and parameter of exponential distribution, by using bayesian approach.

Balakrishnan, Lin and Chan ( 2005) ${ }^{[2]}$ make a comparison of these two prediction intervals based on the expected width of the prediction interval, as well as by means of the probability of the width of one being smaller than the other .Numerical results of these comparisons are presented for different choices of the parameters involved. Also they present an example to illustrate the results discussed in this paper.

Friesl and Hurt (2007) ${ }^{[5]}$ give some basic ideas of both the construction and investigation of the properties of the Bayesian estimates of certain parametric functions of the exponential distribution under the model of random censorship assuming the Koziol-Green model. Various prior distributions are investigated and the corresponding estimates are derived. The stress is put on the asymptotic properties of the estimates with the particular stress on the Bayesian risk. Small sample properties are studied via simulations in the special case.

Liu and Ren (2013) ${ }^{[6]}$ study the empirical Bayes estimation of the parameter of the exponential distribution. In the empirical Bayes procedure, they employ the non-parameter polynomial density estimator to the estimation of the unknown marginal probability density function, instead of estimating the unknown prior probability density function of the parameter. They Empirical derived bayes estimators for the parameter of the exponential distribution under squared error and LINEX loss functions. 


\section{Comparison Bayes Estimators of Reliability in the Exponential Distribution}

They use numerical examples to compare the empirical Bayes estimators obtained under squared error and LINEX loss functions and they get the result of the mean square error of the empirical Bayes estimator under LINEX loss is usually smaller than the estimator under squared error loss function, so it is more better.

So in this paper, we try to find best estimation for Reliability function $(\mathrm{R}(\mathrm{t})$ ) of exponential distribution which it means the probability of surviving at least till age $t$. According to the smallest value of Mean Square Errors (MSE) and Mean Weighted Square Errors (MWSE) were calculated to compare bayes estimators under four types of prior distributions to get bayes estimation :Inverse Chi-square distribution, Inverted Gamma distribution, Improper distribution, Non-informative distribution when the Bayesian estimation based on squared and weighted squared error loss functions .Several cases from exponential distribution for data generating ,for different sample sizes (small, medium, and large).The results were obtained by using simulation technique, Programs written using MATLAB-R2008a program were used.

\section{Exponential Distribution}

We consider $t_{1}, t_{2}, \ldots, t_{n}$ is a random sample of $n$ independent observations from an Exponential distribution having the probability density function (pdf) define as ${ }^{[3]}$ :

$$
\mathrm{f}(\mathrm{t} ; \theta)=\theta^{-1} \exp \left(-\frac{\mathrm{t}}{\theta}\right) \quad, \quad \mathrm{t}>0
$$

where $\theta>0$ is mean, standard deviation, and scale parameter of the distribution, $\theta$ is a survival parameter in the sense that if a random variable $t$ is the duration of time that a given biological or mechanical system manages to survive, and $t \sim \operatorname{Exp}(\theta)$ then the expected duration of survival of the system is $\theta$ units of time .So the cumulative (distribution) function is

$$
\mathrm{F}(\mathrm{t})=\int_{0}^{t} \mathrm{f}(\mathrm{u}) \mathrm{du}=1-\exp \left(-\frac{\mathrm{t}}{\theta}\right) \quad, \quad \mathrm{t}>0
$$

Also, the Reliability function is

$$
\mathrm{R}(\mathrm{t})=1-\mathrm{F}(\mathrm{t})=\int_{t}^{\infty} \mathrm{f}(\mathrm{u}) \mathrm{du}=\exp \left(-\frac{\mathrm{t}}{\theta}\right)
$$

Where $R(t)$ is probability of surviving at least till age $t$.And $F(t)$ is the cumulative distribution function. 


\section{Comparison Bayes Estimators of Reliability in the Exponential Distribution}

\section{Bayes Estimation Method}

In this section, we used several methods to estimation Reliability function( $R(t))$.Let $\underline{t}=\left(t_{1}, t_{2}, \ldots, t_{n}\right)$ be a random sample of size $n$ with probability density function given in equation (1) and likelihood function from the Exponential pdf given in (1) will be as follows ${ }^{[3]}$ :

$$
\mathrm{L}(\underline{\mathrm{t}} \backslash \theta)=\prod_{\mathrm{i}=1}^{\mathrm{n}} \mathrm{f}(\mathrm{t} ; \theta)=\theta^{-\mathrm{n}} \exp \left(-\frac{\sum_{\mathrm{i}=1}^{\mathrm{n}} \mathrm{t}_{\mathrm{i}}}{\theta}\right)
$$

In this paper the posterior distributions for the unknown parameter $\theta$ are derived using the following four types of priors, and then get bayes estimation ${ }^{[3]}$ :

1.Inverse Chi-square distribution ${ }^{[9]}$.

2.Inverted Gamma distribution ${ }^{[8]}$.

3.Improper distribution.

4.Non-informative distribution.

\subsection{The posterior distribution using different Priors}

It is assumed that $\theta$ follows four types of prior distributions with pdf as given in table below:

Table -1: The four types of prior distributions $(P(\theta))$ with pdf for $\theta$.

\begin{tabular}{|c|c|}
\hline Prior distribution & $\mathrm{P}(\theta)$ \\
\hline$\theta \sim$ Inverse Chi-square( v ) & $\mathrm{P}(\theta) \alpha \quad \frac{1}{2^{\frac{\mathrm{v}}{2}}} \theta^{-\frac{\mathrm{v}}{2}-1} \exp \left(-\frac{1}{2 \theta}\right)$ for $\mathrm{v}, \theta>0$ \\
\hline$\theta \sim \operatorname{Inverted} \operatorname{Gamma}(\alpha, \beta)$ & $\mathrm{P}(\theta) \alpha \frac{\beta^{\alpha}}{\Gamma \alpha} \theta^{-(\alpha+1)} \exp \left(-\frac{\beta}{\theta}\right)$ for $\alpha, \beta, \theta>0$ \\
\hline$\theta \sim \operatorname{Improper}(\mathrm{a}, \mathrm{b})$ & $\begin{array}{r}\mathrm{P}(\theta) \alpha \theta^{-(\mathrm{a}+1)} \exp \left(-\frac{\mathrm{b}}{\theta}\right) \text { for } \quad \mathrm{b}, \theta>0 \\
\text { and }-\infty<\mathrm{a}<\infty\end{array}$ \\
\hline$\theta \sim$ Non-informative( $\mathrm{c}$ ) & $\mathrm{P}(\theta) \propto \frac{1}{\theta^{\mathrm{c}}}$ for $\theta, \mathrm{c}>0$ \\
\hline
\end{tabular}

Then the posterior distribution of given the data $\underline{t}=\left(t_{1}, t_{2}, \ldots, t_{n}\right)$ is:

$$
\mathrm{P}(\theta \backslash \mathrm{t})=\frac{\mathrm{L}(\underline{\mathrm{t}} \backslash \theta) \mathrm{P}(\theta)}{\int_{\theta}^{\mathrm{L}(\underline{\mathrm{t}} \backslash \theta)} \mathrm{P}(\theta) \mathrm{d} \theta}
$$

\begin{tabular}{|c|c|}
\hline 4 & 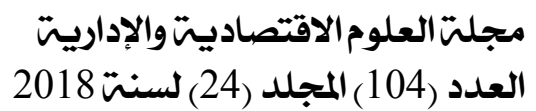 \\
\hline
\end{tabular}




\section{Comparison Bayes Estimators of Reliability in the Exponential Distribution}

Substituting the equation (4) and for each $P(\theta)$ as shown in table -1 in equation (9), we get the posterior distributions for the unknown parameter $\theta$ are derived using the following four types of priors ( for more details see AppendixA).

Table -2: The posterior distributions $(P(\theta \backslash t))$ for the unknown parameter

$(\theta)$ are derived using the following four types of priors.

\begin{tabular}{|c|c|}
\hline Prior dist" & The posterior distribution $(\mathrm{P}(\theta \backslash \mathrm{t}))$ \\
\hline $\begin{array}{l}\text { Inverse } \\
\text { Chi-square }\end{array}$ & 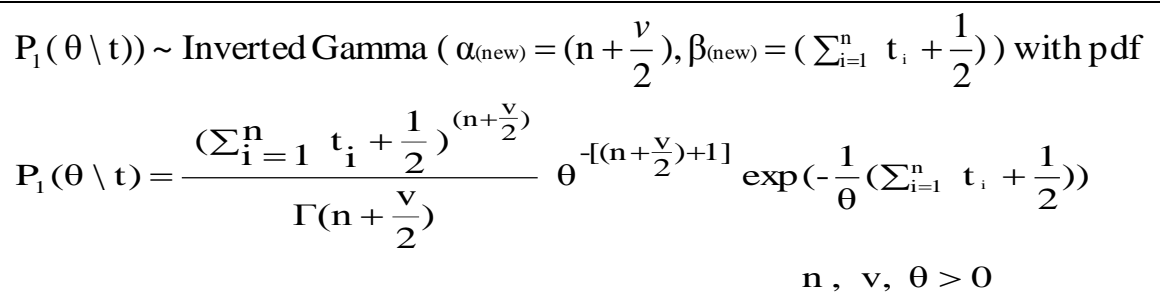 \\
\hline \multirow[t]{2}{*}{$\begin{array}{l}\text { Inverted } \\
\text { Gamma }\end{array}$} & 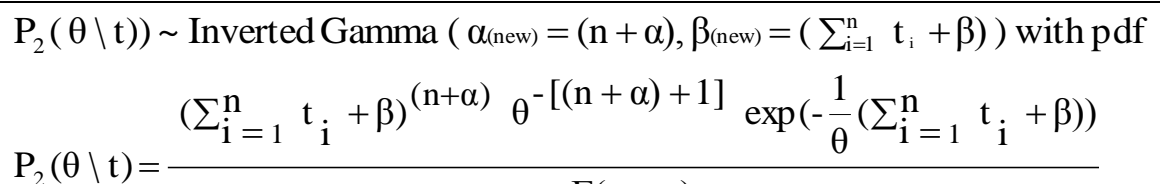 \\
\hline & $\mathrm{n}, \beta, \alpha, \theta>0$ \\
\hline \multirow[t]{2}{*}{ Improper } & $\begin{aligned}\left.\mathrm{P}_{3}(\theta \backslash \mathrm{t})\right) \sim & \text { Inverted Gamma }\left(\alpha_{(\text {new })}=(\mathrm{n}+\mathrm{a}), \beta_{(\text {new })}=\left(\sum_{\mathrm{i}=1}^{\mathrm{n}} \mathrm{t}_{\mathrm{i}}+\mathrm{b}\right)\right) \text { with } \mathrm{pdf} \\
& \left(\sum_{\mathrm{i}=1}^{\mathrm{n}} \mathrm{x}_{\mathrm{i}}+\mathrm{b}\right)^{(\mathrm{n}+\mathrm{a})} \theta^{-[(\mathrm{n}+\mathrm{a})+1]} \exp \left(-\frac{1}{\theta}\left(\sum_{\mathrm{i}=1}^{\mathrm{n}} \mathrm{x}_{\mathrm{i}}+\mathrm{b}\right)\right)\end{aligned}$ \\
\hline & $\begin{array}{c}\Gamma(\mathrm{n}+\mathrm{a}) \\
\mathrm{n}, \mathrm{b}, \theta>0 \text { and }-\infty<\mathrm{a}<\infty\end{array}$ \\
\hline \multirow[t]{2}{*}{$\begin{array}{l}\text { Non- } \\
\text { informative }\end{array}$} & $\begin{array}{l}\left.\mathrm{P}_{4}(\theta \backslash \mathrm{t})\right) \sim \text { Inverted Gamma }\left(\alpha_{(\text {new })}=(\mathrm{n}+\mathrm{c}-1), \beta_{(\text {new })}=\left(\sum_{\mathrm{i}=1}^{\mathrm{n}} \mathrm{t}_{\mathrm{i}}\right)\right) \text { with pdf } \\
\left.\mathrm{P}(\theta \backslash \mathrm{t})=\sum_{\mathrm{i}=1}^{\mathrm{n}} \mathrm{t}_{\mathrm{i}}\right)^{(\mathrm{n}+\mathrm{c}-1)} \theta^{-[(\mathrm{n}+\mathrm{c}-1)+1]} \exp \left(-\frac{1}{\theta} \sum_{\mathrm{i}=1}^{\mathrm{n}} \mathrm{t}_{\mathrm{i}}\right)\end{array}$ \\
\hline & $\Gamma(\mathrm{n}+\mathrm{c}-1)$ \\
\hline
\end{tabular}

\subsection{Bayes' Estimators}

Bayes' estimators for Reliability function $(R=R(t))$, was considered with four different priors and under two loss functions:

1.The squared error loss function $L_{1}(\hat{R}, R)=(\hat{R}-R)^{2}$. 


\section{Comparison Bayes Estimators of Reliability in the Exponential Distribution}

$\begin{array}{lllll}\text { 2.The weighted } & \text { squared } & \text { error } & \text { loss } & \text { function }\end{array}$
$\mathrm{L}_{2}(\hat{\mathrm{R}}, \mathrm{R})=\frac{\mathrm{K}(\hat{\mathrm{R}}-\mathrm{R})^{2}}{\mathrm{R}} \quad$, for $\mathrm{k} \geq 1$.

Where $R$ an estimator for is $R$, was considered with different four priors, and under two loss functions. Following is the derivation of these estimators:

\subsubsection{The squared error loss function}

To obtain the Bayes' estimator, we minimize the posterior expected loss given by:

$\mathrm{L}_{1}(\hat{\mathrm{R}}, \mathrm{R})=(\hat{\mathrm{R}}-\mathrm{R})^{2}$

After simplified steps, we get Bayes estimator of $R(t)$ denoted by $\hat{R}_{S E}(t)$ for the above prior as follows

$$
\hat{\mathrm{R}}_{\mathrm{SE}}=\mathrm{E}(\mathrm{R} \backslash \mathrm{t})=\int_{0}^{\infty} \mathrm{R}(\mathrm{t}) \mathrm{P}(\theta \backslash \mathrm{t}) \mathrm{d} \theta
$$

So, the following results are the derivations of these estimators under the squared error loss function with different four priors (for more details see Appendix-B).

Table -3: The estimators $\left(\hat{R}_{\mathrm{SE}}(t)\right)$ under the squared error loss function with different four priors.

\begin{tabular}{|l|l|}
\hline Prior distribution & $\hat{\mathrm{R}}_{\mathrm{SEi}}(\mathrm{t})=\mathrm{E}(\mathrm{R} \backslash \mathrm{t})=\int_{0}^{\infty} \mathrm{R}(\mathrm{t}) \mathrm{P}(\theta \backslash \mathrm{t}) \mathrm{d} \theta$ \\
\hline $\begin{array}{l}\text { Inverse } \\
\text { square }\end{array}$ & $\hat{\mathrm{R}}_{\mathrm{SE} 1}(\mathrm{t})=\left[\frac{\sum_{\mathrm{i}=1}^{\mathrm{n}} \mathrm{t}_{\mathrm{i}}+0.5}{\sum_{\mathrm{i}=1}^{\mathrm{n}} \mathrm{t}_{\mathrm{i}}+\mathrm{t}+0.5}\right]^{\left(\mathrm{n}+\frac{\mathrm{v}}{2}\right)}, \mathrm{n} \& \mathrm{v}>0$ \\
\hline Inverted Gamma & $\hat{\mathrm{R}}_{\mathrm{SE} 2}(\mathrm{t})=\left[\frac{\sum_{\mathrm{i}=1}^{\mathrm{n}} \mathrm{t}_{\mathrm{i}}+\beta}{\sum_{\mathrm{i}=1}^{\mathrm{n}} \mathrm{t}_{\mathrm{i}}+\mathrm{t}+\beta}\right]^{(\mathrm{n}+\alpha)}, \mathrm{n}, \beta, \alpha>0$ \\
\hline Improper & $\hat{\mathrm{R}}_{\mathrm{SE3}}(\mathrm{t})=\left[\frac{\sum_{\mathrm{i}=1}^{\mathrm{n}} \mathrm{t}_{\mathrm{i}}+\mathrm{b}}{\sum_{\mathrm{i}=1}^{\mathrm{n}} \mathrm{t}_{\mathrm{i}}+\mathrm{t}+\mathrm{b}}\right]^{(\mathrm{n}+\mathrm{a})}, \mathrm{n}, \mathrm{b}, \mathrm{a}>0$ \\
\hline Non-informative & $\hat{\mathrm{R}}_{\mathrm{SE} 4}(\mathrm{t})=\left[\frac{\sum_{\mathrm{i}=1}^{\mathrm{n}} \mathrm{t}_{\mathrm{i}}}{\sum_{\mathrm{i}=1}^{\mathrm{n}} \mathrm{t}_{\mathrm{i}}+\mathrm{t}}\right]^{(\mathrm{n}+\mathrm{c}-1)}, \mathrm{n}, \mathrm{c}>0$ \\
\hline
\end{tabular}

6

مجلتة العلوم الاقتصاديت والإداريت

العدد (104) المجلد (24) لسنت 2018 


\section{Comparison Bayes Estimators of Reliability in the Exponential Distribution}

\subsubsection{The weighted squared error loss function}

To obtain the Bayes' estimator, we minimize the posterior expected loss given by:

$\mathrm{L}_{2}(\hat{\mathrm{R}}, \mathrm{R})=\frac{\mathrm{K}(\hat{\mathrm{R}}-\mathrm{R})^{2}}{\mathrm{R}} \quad$, for $\mathrm{k} \geq 1$

After simplified steps, we get Bayes estimator of $R(t)$ denoted by $\hat{R}_{w S E}(t)$ for the above prior as follows

$$
\hat{\mathrm{R}}_{\mathrm{WSE}}=\frac{1}{\mathrm{E}\left(\frac{1}{\mathrm{R}} \backslash \mathrm{x}\right)}=\frac{1}{\int_{0}^{\infty} \frac{1}{\mathrm{R}} \mathrm{P}(\theta \backslash \mathrm{t}) \mathrm{d} \theta}
$$

So, the following results are the derivations of these estimators under the weighted squared error loss function with different six priors (for more details see Appendix-C).

Table -4: The estimators $\left(\hat{R}_{\mathrm{WSE}}(\mathrm{t})\right)$ under the weighted squared error loss function with different four priors.

\begin{tabular}{|l|l|}
\hline Prior distribution & $\hat{\mathrm{R}}_{\mathrm{WSE}}(\mathrm{t})=\frac{1}{\mathrm{E}\left(\frac{1}{\mathrm{R}} \backslash \mathrm{x}\right)}=\frac{1}{\int_{0}^{\infty} \frac{1}{\mathrm{R}} \mathrm{P}(\theta \backslash \mathrm{t}) \mathrm{d} \theta}$ \\
\hline Inverse Chi-square & $\hat{\mathrm{R}}_{\mathrm{WSE} 1}(\mathrm{t})=\left[\frac{\sum_{\mathrm{i}=1}^{\mathrm{n}} \mathrm{t}_{\mathrm{i}}+0.5}{\sum_{\mathrm{i}=1}^{\mathrm{n}} \mathrm{t}_{\mathrm{i}}-\mathrm{t}+0.5}\right]^{-\left(\mathrm{n}+\frac{\mathrm{v}}{2}\right)}, \mathrm{n} \& \mathrm{v}>0$ \\
\hline Inverted Gamma & $\hat{\mathrm{R}}_{\mathrm{WSE2}}(\mathrm{t})=\left[\frac{\sum_{\mathrm{i}=1}^{\mathrm{n}} \mathrm{t}_{\mathrm{i}}+\beta}{\sum_{\mathrm{i}=1}^{\mathrm{n}} \mathrm{t}_{\mathrm{i}}-\mathrm{t}+\beta}\right]^{-(\mathrm{n}+\alpha)}, \mathrm{n}, \beta, \alpha>0$ \\
\hline Improper & $\hat{\mathrm{R}}_{\mathrm{WSE3}}(\mathrm{t})=\left[\frac{\sum_{\mathrm{i}=1}^{\mathrm{n}} \mathrm{t}_{\mathrm{i}}+\mathrm{b}}{\sum_{\mathrm{i}=1}^{\mathrm{n}} \mathrm{t}_{\mathrm{i}}-\mathrm{t}+\mathrm{b}}\right]^{-(\mathrm{n}+\mathrm{a})}, \mathrm{n}, \mathrm{b}, \mathrm{a}>0$ \\
\hline Non-informative & $\hat{\mathrm{R}}_{\mathrm{WSE4}}(\mathrm{t})=\left[\frac{\sum_{\mathrm{i}=1}^{\mathrm{n}} \mathrm{t}_{\mathrm{i}}}{\sum_{\mathrm{i}=1}^{\mathrm{n}} \mathrm{t}_{\mathrm{i}}-\mathrm{t}}\right]^{-(\mathrm{n}+\mathrm{c}-1)}, \mathrm{n}, \mathrm{c}>0$ \\
\hline
\end{tabular}

\section{Simulation Study}

In this study, we have generated random samples from Exponential distribution and compared the performance of Bayes estimators based on them. So we have considered several steps to perform simulation study as follow:

1.We have chosen sample size $n=30,60,90$ and 120 to represent small, moderate and large sample size. 


\section{Comparison Bayes Estimators of Reliability in the Exponential Distribution}

2.We generated data from Exponential distribution for the scale parameter; we have considered randomly several values for the parameter of Exponential distribution $\theta=0.5,1,1.5,2.5$.

3.We choose the values for the parameters of the prior distributions that give the appropriate estimation for Reliability function $(R(t))$, as shown below:

- We used two values for the parameter of the Inverse Chi-square distribution $(v=4,6)$ as prior distribution for $\theta$.

- We used two values for the parameters of the Inverted Gamma distribution $(\alpha, \beta)=((3,1),(5,2))$ as prior distribution for $\theta$.

- We used two values for the parameters of the improper distribution $(\mathrm{a}, \mathrm{b})=((7,3),(9,3))$ as prior distribution for $\theta$.

- We used randomly three values for the function of the non-informative prior distribution $\mathrm{c}=\mathbf{1}, 3$.

4. The number of replication used was $(L=1000)$ for each sample size $(n)$.

5. The true $R(t)$ is computed according to the formula (3) with $\theta=0.5,1,1.5,2.5$ and the true $\mathrm{t}$ is $\mathrm{t}=0.5,1.5,2.5,3.5$.

6. We obtained estimators for Reliability function $(R(t))$, the estimators in table $(3)$, it means the estimators $\left(R_{S E}(t)\right)$ under the squared error loss function with four different priors .And the estimators in table (4), it means the estimators $\left(\mathrm{R}_{\mathrm{WSE}}(\mathrm{t})\right.$ ) under the weighted squared error loss function with different four priors.

The simulation program was written by using MATLAB-R2008a program. After the Reliability function $(\mathrm{R}(\mathrm{t})$ ), was estimated, Mean Square Errors (MSE) and Mean weighted squared Errors (MWSE) were calculated to compare between the bayes estimators, where $k=1$. So we have the following criteria:

$$
\begin{aligned}
& \operatorname{MSE}(\hat{R}(t))=\frac{1}{L} \sum_{L=1}^{1000}\left(\hat{R}_{L}(t)-R(t)\right)^{2} \\
& \operatorname{MWSE}(\hat{R}(t))=\frac{1}{L} \sum_{L=1}^{1000}\left(\frac{\left.\hat{R_{L}}(t)-R(t)\right)^{2}}{R(t)}\right)
\end{aligned}
$$

See appendix-D, for the programs algorithm. The results of the simulation study are summarized and tabulated in tables (4-1to4-4).In each row of tables (4-1to4-4) ,we have four estimated values for $R(t)(R(t))$ with MSE for all sample size (n) and values $(\mathrm{v},(\alpha, \beta),(\mathrm{a}, \mathrm{b}), \mathrm{c})$ respectively. Also the results of the simulation study are summarized and tabulated in tables (4-5to4-8).In each row of tables (4-5to4-8), we have four estimated values for $R(t)(R(t))$ with MWSE for all sample size (n) and values $(v,(\alpha, \beta),(\mathbf{a}, \mathbf{b}), \mathbf{c})$ respectively .The Bayes estimators under four types of prior distribution .So our criteria is the best estimator that gives the smallest value of ( MSE ) and (MWSE ). We list the results in the following tables (4-1 to4-8). 


\section{Comparison Bayes Estimators of Reliability in the Exponential Distribution}

Table 4-1: Reliability Estimation using Bayes with respect to

$\operatorname{MSE}(\mathrm{R}(\mathrm{t})$ ) when prior distribution is inverse Chi-square .

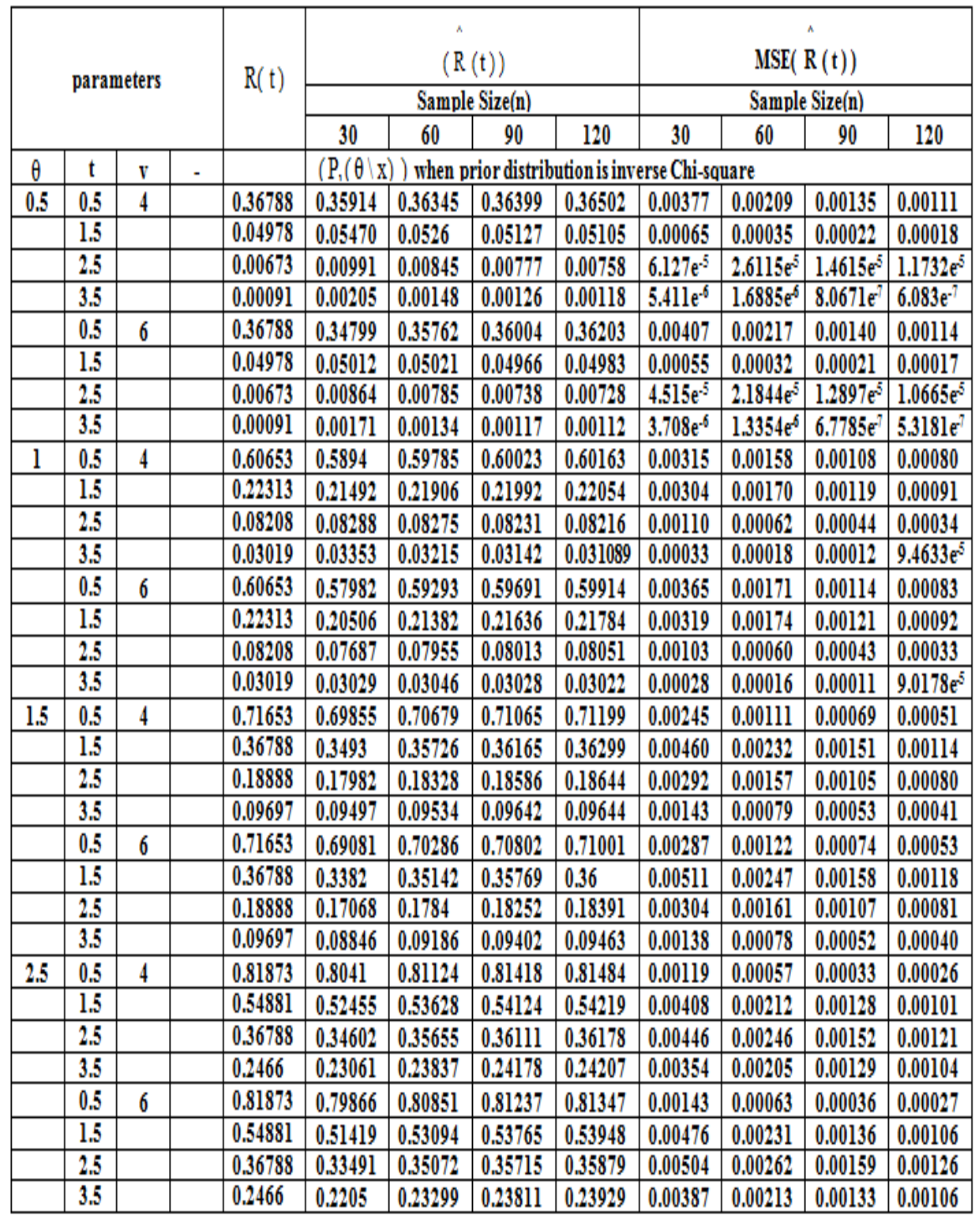




\section{Comparison Bayes Estimators of Reliability in the Exponential Distribution}

Table4- 2: Reliability Estimation using Bayes with respect to

$\operatorname{MSE}(\mathrm{R}(\mathrm{t})$ ) when prior distribution is inverted gamma .

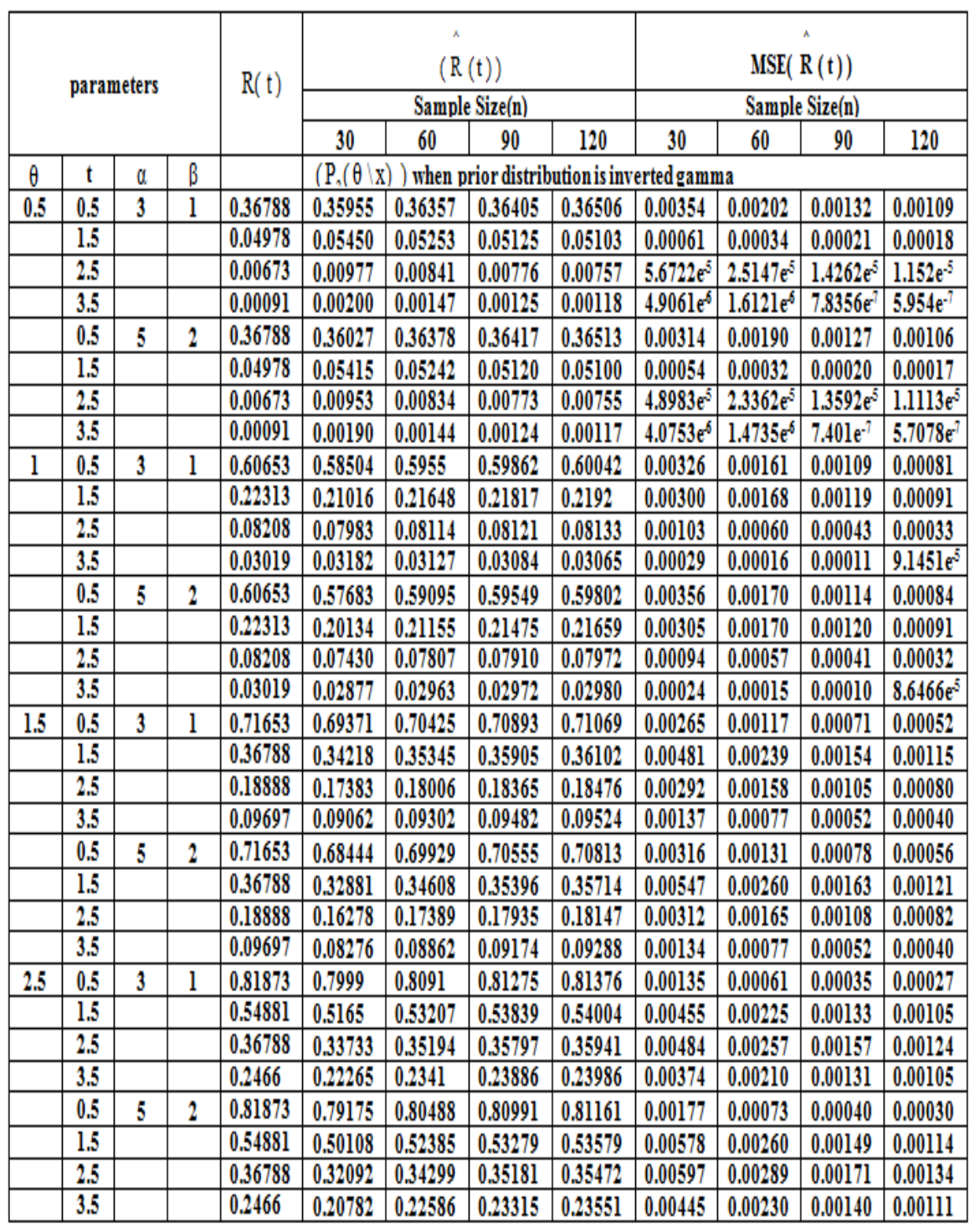




\section{Comparison Bayes Estimators of Reliability in the Exponential Distribution}

Table4- 3: Reliability Estimation using Bayes with respect to $\wedge$

$\operatorname{MSE}(\mathrm{R}(\mathrm{t})$ ) when prior distribution is improper .

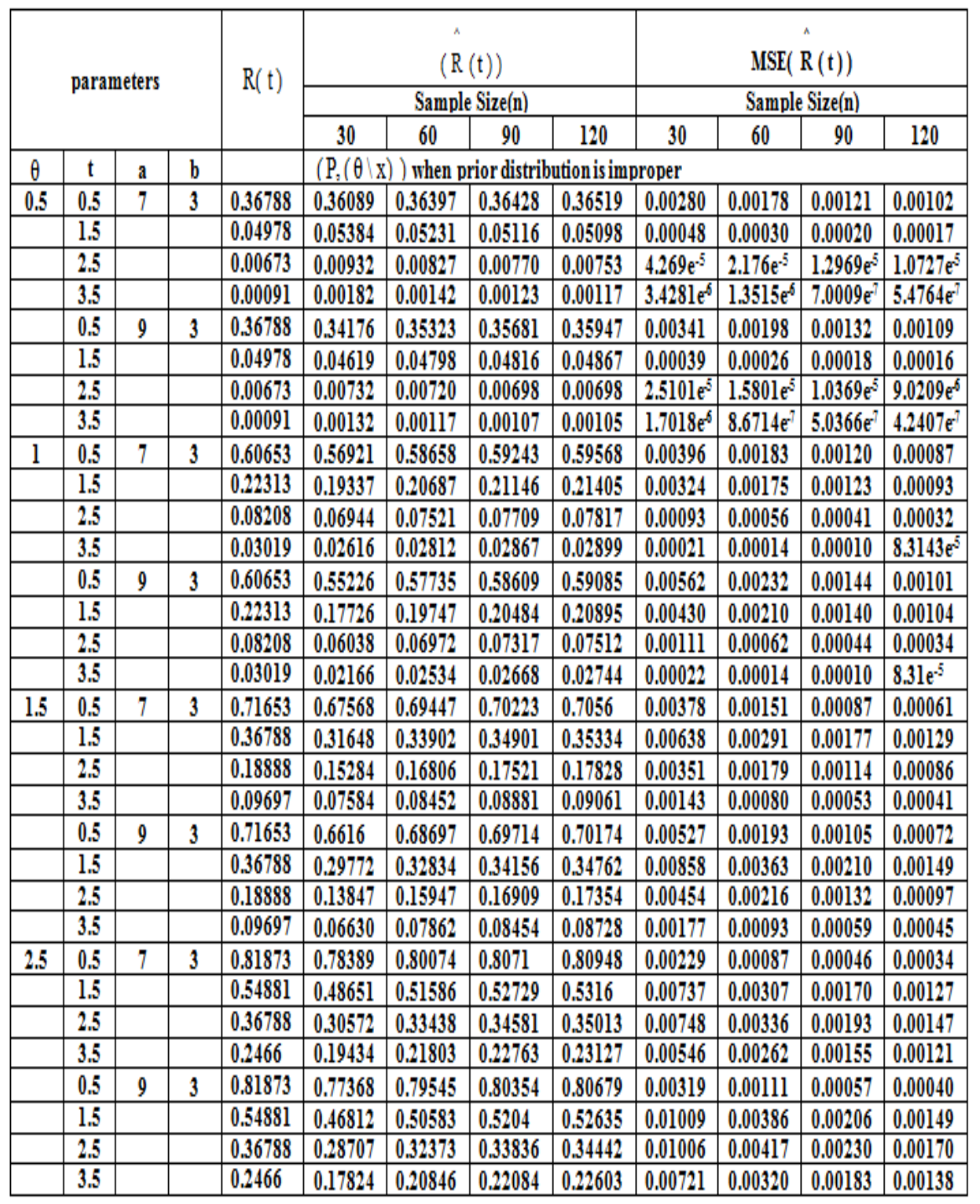




\section{Comparison Bayes Estimators of Reliability in the Exponential Distribution}

Table4- 4: Reliability Estimation using Bayes with respect to

$\operatorname{MSE}(\mathrm{R}(\mathrm{t}))$ when prior distribution is non -informative .

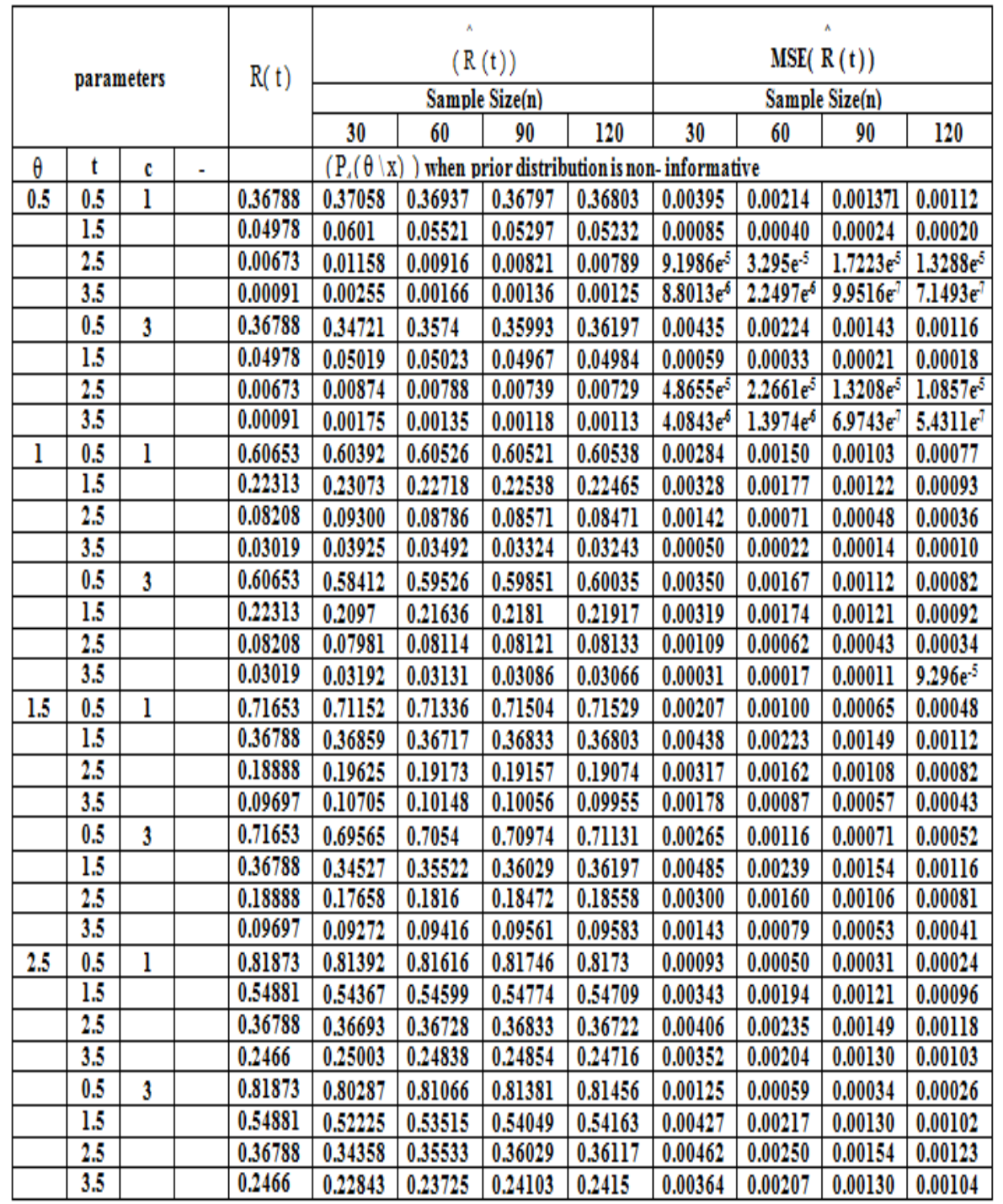




\section{Comparison Bayes Estimators of Reliability in the Exponential Distribution}

Table4-5: Reliability Estimation using Bayes with respect to

$\operatorname{MWSE}(\mathrm{R}(\mathrm{t}))$ when prior distribution is inverse Chi-square .

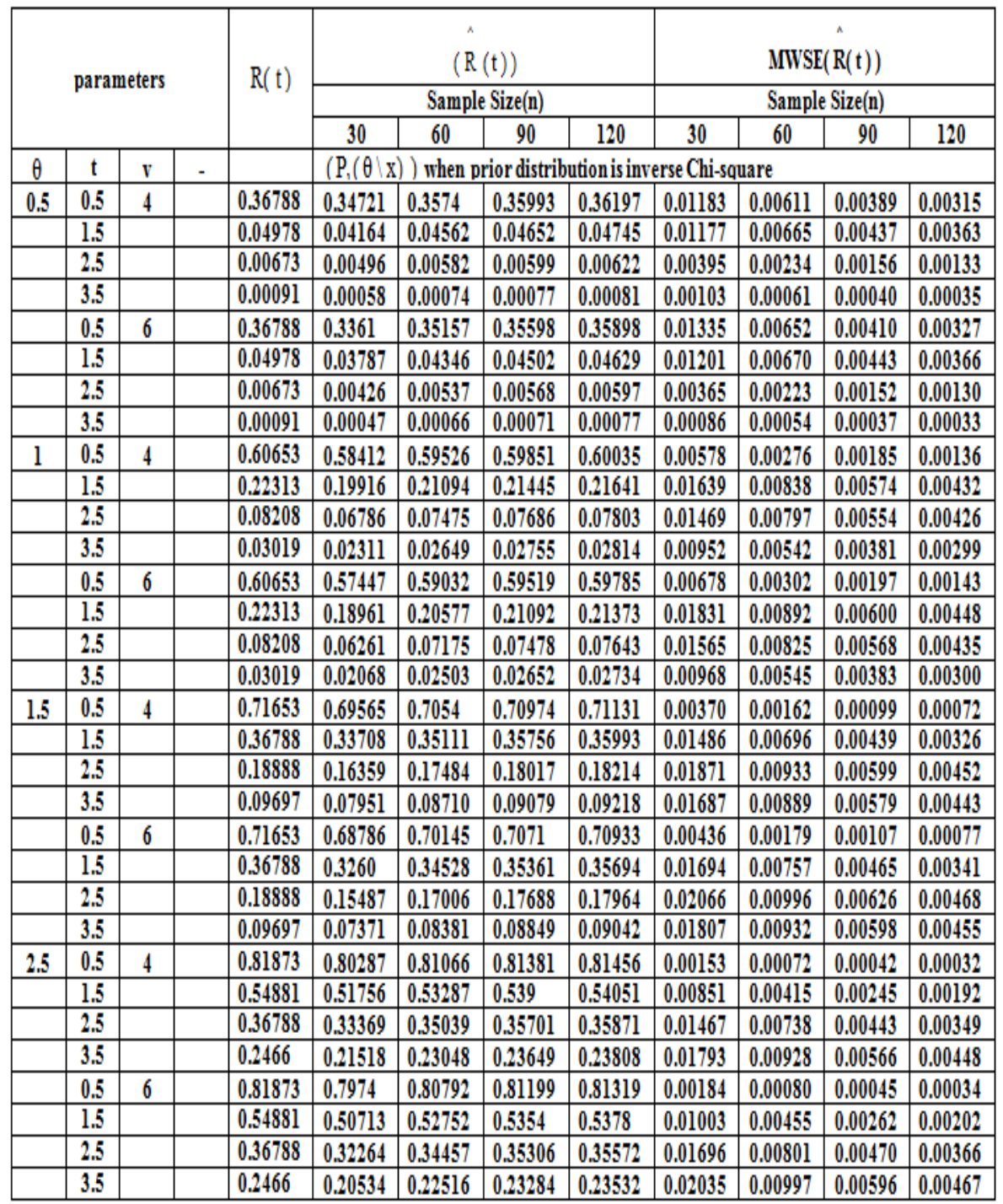




\section{Comparison Bayes Estimators of Reliability in the Exponential Distribution}

Table4-6: Reliability Estimation using Bayes with respect to $\wedge$

$\operatorname{MWSE}(\mathrm{R}(\mathrm{t})$ ) when prior distribution is inverted gamma.

\begin{tabular}{|c|c|c|c|c|c|c|c|c|c|c|c|c|}
\hline \multirow{2}{*}{\multicolumn{4}{|c|}{ parameters }} & \multirow{4}{*}{$R(t)$} & \multirow{2}{*}{\multicolumn{4}{|c|}{$\frac{(\mathrm{R}(\mathrm{t}))}{\text { Sample Size(n) }}$}} & \multirow{2}{*}{\multicolumn{4}{|c|}{$\begin{array}{l}\hat{\operatorname{MWSE}}(\mathrm{R}(\mathrm{t})) \\
\text { Sample Size(n) }\end{array}$}} \\
\hline & & & & & & & & & & & & \\
\hline & & & & & 30 & 60 & 90 & 120 & 30 & 60 & 90 & 120 \\
\hline$\theta$ & $\mathrm{t}$ & $a$ & $B$ & & \multicolumn{8}{|c|}{$\left(\mathrm{P}_{n}(\theta \backslash \mathrm{x})\right)$ when prior distribution is inverted gamma } \\
\hline \multirow[t]{8}{*}{0.5} & 0.5 & 3 & 1 & 0.36788 & 0.34799 & 0.35762 & 0.36004 & 0.36203 & 0.01108 & 0.00591 & 0.00380 & 0.00310 \\
\hline & 1.5 & & & 0.04978 & 0.04179 & 0.04567 & 0.04655 & 0.04746 & 0.01116 & 0.00645 & 0.00428 & 0.00358 \\
\hline & 2.5 & & & 0.00673 & 0.00497 & 0.00582 & 0.00600 & 0.00622 & 0.00374 & 0.00227 & 0.00152 & 0.00131 \\
\hline & 3.5 & & & 0.00091 & 0.00058 & 0.00074 & 0.00077 & 0.00081 & 0.00096 & 0.00059 & 0.00039 & 0.00035 \\
\hline & 0.5 & 5 & 2 & 0.36788 & 0.3494 & 0.35802 & 0,36024 & 0.36215 & 0.00978 & 0.00554 & 0.00364 & 0.00300 \\
\hline & 1.5 & & & 0.04978 & 0.04209 & 0.04575 & 0.04660 & \begin{tabular}{|l|l}
0.04749 \\
\end{tabular} & 0.01008 & 0.00609 & 0.00411 & 0.00347 \\
\hline & 2.5 & & & 0.00673 & 0.00499 & 0.00582 & 0.00600 & 0.00622 & 0.00338 & 0.00215 & 0.00146 & 0.001271 \\
\hline & 3.5 & & & 0.00091 & 0.00058 & 0.00073 & 0.00077 & 0.00081 & 0.00086 & 0.00055 & 0.00037 & 0.00034 \\
\hline \multirow[t]{8}{*}{1} & 0.5 & 3 & 1 & 0.60653 & 0.57982 & 0.59293 & 0.59691 & 0.59914 & 0.00602 & 0.00283 & 0.00188 & 0.00138 \\
\hline & 1.5 & & & 0.22313 & 0.19478 & 0.20846 & 0.21274 & 0.2151 & 0.01674 & 0.00849 & 0.00579 & 0.00435 \\
\hline & 2.5 & & & 0.08208 & 0.06538 & 0.07329 & 0.07584 & 0.07724 & 0.01471 & 0.00796 & 0.00554 & \\
\hline & 3.5 & & & 0.03019 & 0.02194 & 0.02577 & 0.02704 & \begin{tabular}{|l}
0.02774 \\
\end{tabular} & 0.00932 & 0.00534 & 0.00377 & 0.00297 \\
\hline & 0.5 & 5 & 2 & 0.60653 & 0.57172 & 0.5884 & 0.59379 & 0.59675 & 0.00663 & 0.00301 & 0.00198 & 0.00144 \\
\hline & 1.5 & & & 0.22313 & 0.1867 & 0.20372 & 0.20941 & 0.21254 & 0.01787 & 0.00885 & 0.00598 & 0.00447 \\
\hline & 2.5 & & & 0.08208 & 0.06091 & 0.07053 & 0.07387 & 0.07571 & 0.01519 & 0.00811 & 0.00562 & 0.00431 \\
\hline & 3.5 & & & 0.03019 & 0.01986 & 0,02442 & 0.02606 & \begin{tabular}{|l}
0.02698 \\
\end{tabular} & 0.00929 & 0.00531 & 0.00376 & 0.00296 \\
\hline \multirow[t]{8}{*}{1.5} & 0.5 & 3 & 1 & 0.71653 & 0.69081 & 0.70286 & 0.70802 & 0.71001 & 0.00401 & 0.00171 & 0.00103 & 0.00075 \\
\hline & 1.5 & & & 0.36788 & 0.33012 & 0,34734 & 0.35498 & 0.35796 & 0.01582 & 0.00727 & 0.00452 & 0.00334 \\
\hline & 2.5 & & & 0.18888 & 0.15804 & 0.17173 & 0.17801 & 0.18049 & 0.01955 & 0.00962 & 0.00612 & 0.00459 \\
\hline & 3.5 & & & 0.09697 & 0.07577 & 0.08495 & 0.08927 & 0.09102 & 0.01730 & 0.00906 & 0.00586 & 0.00448 \\
\hline & 0.5 & 5 & 2 & 0.71653 & 0.68155 & 0.69789 & 0.70463 & 0.70745 & 0.00479 & 0.00193 & 0.00113 & 0.00080 \\
\hline & 1.5 & & & 0.36788 & 0.31708 & 0,34005 & 0.34992 & 0.3541 & 0.01832 & 0.00807 & 0.00488 & 0.00355 \\
\hline & 2.5 & & & 0.18888 & 0.14781 & 0.16578 & 0.17381 & 0.17726 & 0.02197 & 0.01047 & 0.00650 & 0.00483 \\
\hline & 3.5 & & & 0.09697 & 0.06903 & 0.08087 & 0.08634 & 0.08875 & 0.01887 & 0.00966 & 0.00614 & 0.00465 \\
\hline \multirow[t]{8}{*}{2.5} & 0.5 & 3 & 1 & 0.81873 & 0.79866 & 0.80851 & 0.81237 & 0.81347 & 0.00174 & 0.00077 & 0.00044 & 0.00034 \\
\hline & 1.5 & & & 0.54881 & 0.50951 & 0.52867 & 0.53615 & 0.53836 & 0.00955 & 0.00443 & 0.00257 & 0.00199 \\
\hline & 2.5 & & & 0.36788 & 0.32512 & 0,34581 & 0.35388 & 0.35634 & 0.01623 & 0.00781 & 0.00462 & 0.00361 \\
\hline & 3.5 & & & 0.2466 & 0.20752 & 0.22628 & 0.2336 & 0.23589 & 0.01957 & 0.00975 & 0.00587 & $0.0046 \mathrm{l}$ \\
\hline & 0.5 & 5 & 2 & 0.81873 & 0.79048 & 0.80429 & 0.80952 & 0.81132 & 0.00228 & 0.00092 & 0.00050 & 0.00037 \\
\hline & 1.5 & & & 0.54881 & 0.49409 & 0.52044 & 0.53055 & 0.53411 & 0.01219 & 0.00517 & 0.00289 & 0.00219 \\
\hline & 2.5 & & & 0.36788 & 0.30898 & 0.33691 & 0,34775 & 0.35166 & 0.02024 & 0.00898 & 0.00515 & 0.00394 \\
\hline & 3.5 & & & 0.2466 & 0.19331 & 0.2182 & 0.22796 & 0.23157 & 0.02384 & 0.01104 & 0.00647 & 0.00499 \\
\hline
\end{tabular}




\section{Comparison Bayes Estimators of Reliability in the Exponential Distribution}

Table4-7: Reliability Estimation using Bayes with respect to $\wedge$

$\operatorname{MWSE}(\mathrm{R}(\mathrm{t})$ ) when prior distribution is improper .

\begin{tabular}{|c|c|c|c|c|c|c|c|c|c|c|c|c|}
\hline \multirow{3}{*}{\multicolumn{4}{|c|}{ parameters }} & \multirow{4}{*}{$R(t)$} & \multicolumn{4}{|c|}{$\hat{(R(t))}$} & \multicolumn{4}{|c|}{$\hat{\operatorname{MWSE}}(\hat{R}(\mathrm{t}))$} \\
\hline & & & & & \multicolumn{4}{|c|}{ Sample Size(n) } & \multicolumn{4}{|c|}{ Sample Size(n) } \\
\hline & & & & & 30 & 60 & 90 & 120 & 30 & 60 & 90 & 120 \\
\hline$\theta$ & $t$ & $a$ & $\mathrm{~b}$ & & \multicolumn{8}{|c|}{$(P,(\theta \backslash \mathrm{X}))$ when prior distribution is improper } \\
\hline \multirow[t]{8}{*}{0.5} & 0.5 & 7 & 3 & 0.36788 & 0,35063 & 0,35839 & 0.36043 & 0,36226 & 0.00870 & 0.00520 & 0.00349 & 0.00290 \\
\hline & 1.5 & & & 0.04978 & 0.04238 & 0.04584 & 0.04665 & 0.04751 & 0.00914 & 0.00575 & 0.00395 & 0.00336 \\
\hline & 2.5 & & & 0.00673 & 0.00502 & 0.00583 & 0.00601 & 0.00623 & 0.00308 & 0.00203 & 0.00141 & 0.00123 \\
\hline & 3.5 & & & 0.00091 & 0.00058 & 0.00073 & 0.00077 & 0.00081 & 0.00077 & 0.00052 & 0.00036 & 0.00032 \\
\hline & 0.5 & 9 & 3 & 0.36788 & 0.33155 & 0.34765 & 0.35296 & 0.35654 & 0.01140 & 0.00605 & 0.00394 & 0.00316 \\
\hline & 1.5 & & & 0.04978 & 0.03595 & 0.04189 & 0.04383 & 0.04531 & 0.01026 & 0.00606 & 0.00416 & 0.00346 \\
\hline & 2.5 & & & 0.00673 & 0.00383 & 0.00502 & 0.00542 & 0.00576 & 0.00297 & 0.00194 & 0.00138 & 0.00120 \\
\hline & 3.5 & & & 0.00091 & 0.00040 & 0.00060 & 0,00066 & 0.00073 & 0,00063 & 0.00045 & 0.00033 & 0.00030 \\
\hline \multirow[t]{8}{*}{1} & 0.5 & 7 & 3 & 0.60653 & 0.56422 & 0.58406 & 0.59075 & 0.59441 & 0.00738 & 0.00325 & 0.00210 & 0.00151 \\
\hline & 1.5 & & & 0.22313 & 0.1794 & 0.19923 & 0.20621 & 0.21005 & 0,01942 & 0.00938 & 0.00625 & 0.00464 \\
\hline & 2.5 & & & 0.08208 & 0.05698 & 0.06796 & 0.072 & 0.07424 & 0.01609 & 0.00843 & 0.00579 & 0.00442 \\
\hline & 3.5 & & & 0.03019 & 0.01808 & 0.02318 & 0.02514 & 0.02625 & 0.00959 & 0.00541 & 0.00382 & 0.00299 \\
\hline & 0.5 & 9 & 3 & 0.60653 & 0.54716 & 0.5748 & 0.58439 & 0.58957 & 0.01043 & 0.00415 & 0.00253 & 0.00176 \\
\hline & 1.5 & & & 0.22313 & 0.16385 & 0.18998 & 0.19966 & \begin{tabular}{|l}
0.20498 \\
\end{tabular} & 0.02576 & \begin{tabular}{|l}
0.01149 \\
\end{tabular} & 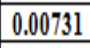 & 0.00528 \\
\hline & 2.5 & & & 0.08208 & 0.04909 & 0.06282 & 0.06825 & 0.07129 & 0.02017 & 0.00995 & 0.00659 & 0.00491 \\
\hline & 3.5 & & & 0.03019 & 0.01472 & 0.02078 & 0,02334 & 0.02480 & 0.01141 & 0.00615 & 0.00422 & 0.00324 \\
\hline \multirow[t]{8}{*}{1.5} & 0.5 & 7 & 3 & 0.71653 & 0.6728 & \begin{tabular}{|l}
0.69307 \\
\end{tabular} & 0.70131 & 0.70492 & 0,00573 & 0.00222 & 0.00126 & 0.00088 \\
\hline & 1.5 & & & 0.36788 & 0.30507 & 0,33307 & 0.345 & 0,35032 & 0.02141 & 0.00911 & 0.00536 & 0.00383 \\
\hline & 2.5 & & & 0.18888 & 0.13864 & 0.16016 & 0.16976 & 0.17412 & 0.02510 & 0.01162 & 0.00705 & 0.00516 \\
\hline & 3.5 & & & 0.09697 & 0.06313 & 0.07707 & 0.08355 & 0.08656 & 0.02108 & 0.01054 & 0.00657 & 0.00491 \\
\hline & 0.5 & 9 & 3 & 0.71653 & 0.65863 & 0.68555 & 0.69621 & 0.70105 & 0.00793 & 0.00284 & 0.00154 & 0.00104 \\
\hline & 1.5 & & & 0.36788 & 0.28646 & 0,32241 & 0.33756 & 0.3446 & 0.02843 & 0.01139 & 0.00642 & 0.00446 \\
\hline & 2.5 & & & 0.18888 & 0.12501 & 0.15177 & 0.16373 & 0.16943 & 0.03212 & 0.01419 & 0.00830 & 0.00591 \\
\hline & 3.5 & & & 0.09697 & 0.05472 & 0.07150 & 0.07944 & 0.08332 & 0.02609 & 0.01257 & 0.00760 & 0.00555 \\
\hline \multirow[t]{8}{*}{2.5} & 0.5 & 7 & 3 & 0.81873 & 0.7826 & 0.80014 & 0.80671 & 0.80919 & 0.00294 & 0.00110 & 0.00058 & 0.00042 \\
\hline & 1.5 & & & 0.54881 & 0.00294 & 0.51245 & 0.52505 & 0.52992 & 0.01544 & 0.00612 & 0,00332 & 0.00245 \\
\hline & 2.5 & & & 0.36788 & 0.29404 & 0,32836 & 0.34177 & 0.34709 & 0.02516 & 0.01050 & 0.00585 & 0.00437 \\
\hline & 3.5 & & & 0.2466 & 0.18042 & 0.21051 & 0.2225 & 0.22737 & 0.02911 & \begin{tabular}{|l}
0.01276 \\
\end{tabular} & \begin{tabular}{|l}
0.00728 \\
\end{tabular} & 0.00550 \\
\hline & 0.5 & 9 & 3 & 0.81873 & 0.77234 & 0.79484 & 0.80315 & 0.8065 & 0.00408 & 0.00141 & 0.00071 & 0.00050 \\
\hline & 1.5 & & & 0.54881 & 0.46108 & 0.50238 & 0.51814 & 0.52466 & 0.02088 & 0.00770 & 0.00404 & 0.00289 \\
\hline & 2.5 & & & 0.36788 & 0.27556 & 0.31773 & 0.33433 & 0.34138 & 0.03321 & 0.01304 & 0.00704 & 0.00510 \\
\hline & 3.5 & & & 0.2466 & 0.16488 & 0.20107 & 0.21577 & 0.22216 & 0.03756 & 0.01562 & 0.00868 & 0.00636 \\
\hline
\end{tabular}




\section{Comparison Bayes Estimators of Reliability in the Exponential Distribution}

Table4-8: Reliability Estimation using Bayes with respect to

$\operatorname{MWSE}(\mathrm{R}(\mathrm{t})$ ) when prior distribution is non -informative.

\begin{tabular}{|c|c|c|c|c|c|c|c|c|c|c|c|c|}
\hline & \multirow{3}{*}{\multicolumn{3}{|c|}{ parameters }} & \multirow{3}{*}{$R(t)$} & \multicolumn{4}{|c|}{$\hat{R}(t))$} & \multicolumn{4}{|c|}{$\hat{\operatorname{MWSE}}(\hat{R}(\mathrm{t}))$} \\
\hline & & & & & \multicolumn{4}{|c|}{ Sample Size(n) } & \multicolumn{4}{|c|}{ Sample Size(n) } \\
\hline & & & & & 30 & 60 & 90 & 120 & 30 & 60 & 90 & 120 \\
\hline$\theta$ & $t$ & c & . & & \multicolumn{8}{|c|}{$\left(P_{1}(\theta \backslash x)\right)$ when prior distribution is non-informative } \\
\hline \multirow[t]{8}{*}{0.5} & 0.5 & 1 & & 0.36788 & 0.35822 & 0.36321 & 0.36387 & 0.36495 & 0.01170 & 0.00607 & 0.00385 & 0.00313 \\
\hline & 1.5 & & & 0.04978 & 0.04574 & 0.04789 & 0.04807 & 0.04863 & 0.01294 & 0.00701 & 0.00449 & 0.00373 \\
\hline & 2.5 & & & 0.00673 & 0.00580 & 0.00630 & 0.00633 & 0.00648 & 0.00487 & 0.00263 & 0.00167 & 0.00141 \\
\hline & 3.5 & & & 0.00091 & 0.00073 & 0.00083 & 0.00083 & 0.00086 & 0.00143 & 0.00073 & 0.00045 & 0.00039 \\
\hline & 0.5 & 3 & & 0.36788 & 0.33492 & 0.35126 & 0.35583 & 0.3589 & 0.01428 & 0.00675 & 0.00419 & 0.00332 \\
\hline & 1.5 & & & 0.04978 & 0.03762 & 0.04339 & 0.04498 & 0.04627 & 0.01262 & 0.00689 & 0.00452 & 0.00371 \\
\hline & 2.5 & & & 0.00673 & 0.00423 & 0.00536 & 0.00567 & 0.00597 & 0.00382 & 0.00229 & 0.00155 & 0.00132 \\
\hline & 3.5 & & & 0.00091 & 0.00047 & 0.00066 & 0.00071 & 0.00077 & 0.00090 & 0.00056 & 0.00038 & 0.00034 \\
\hline \multirow[t]{8}{*}{1} & 0.5 & l & & 0.60653 & 0.59865 & 0.60268 & 0.60349 & 0.6041 & 0.00502 & 0.00256 & 0.00175 & 0.00130 \\
\hline & 1.5 & & & 0.22313 & 0.21425 & 0.21888 & 0.21983 & 0.22048 & 0.01545 & 0.00814 & 0.00560 & 0.00424 \\
\hline & 2.5 & & & 0.08208 & 0.07655 & 0.07948 & 0.08009 & 0.08048 & 0.01504 & 0.00810 & 0.00558 & 0.00428 \\
\hline & 3.5 & & & 0.03019 & 0.02731 & 0.02885 & 0.02918 & 0.02938 & 0.01058 & 0.00576 & 0.00396 & 0.00308 \\
\hline & 0.5 & 3 & & 0.60653 & 0.5787 & 0.59264 & 0.59678 & 0.59906 & 0.00648 & 0.00293 & 0.00193 & 0.00140 \\
\hline & 1.5 & & & 0.22313 & 0.19385 & 0.20821 & 0.21262 & 0.21503 & 0.01780 & 0.00876 & 0.00592 & 0.00443 \\
\hline & 2.5 & & & 0.08208 & 0.06497 & 0.07317 & 0.07578 & 0.07721 & 0.01550 & 0.00820 & 0.00566 & 0.00433 \\
\hline & 3.5 & & & 0.03019 & 0.02179 & 0.02572 & 0.02702 & 0.02773 & 0.00978 & 0.00549 & 0.00385 & 0.00301 \\
\hline \multirow[t]{8}{*}{1.5} & 0.5 & l & & 0.71653 & 0.70868 & 0.71198 & 0.71413 & 0.71461 & 0.00305 & 0.00144 & 0.00092 & 0.00068 \\
\hline & 1.5 & & & 0.36788 & 0.35616 & 0.36098 & 0.36423 & 0.36495 & 0.01306 & 0.00641 & 0.00417 & 0.00313 \\
\hline & 2.5 & & & 0.18888 & 0.17916 & 0.18307 & 0.18578 & 0.18639 & 0.01752 & 0.00889 & 0.00583 & 0.00442 \\
\hline & 3.5 & & & 0.09697 & 0.09019 & 0.09287 & 0.09477 & 0.09520 & 0.01682 & 0.00877 & 0.00577 & 0.00441 \\
\hline & 0.5 & 3 & & 0.71653 & 0.6927 & \begin{tabular}{|l}
0.70399 \\
\end{tabular} & 0.70882 & 0.71063 & 0.00400 & 0.00169 & 0.00102 & 0.00074 \\
\hline & 1.5 & & & 0.36788 & 0.33292 & 0.34904 & 0.35619 & 0.3589 & 0.01584 & 0.00723 & 0.00450 & 0.00332 \\
\hline & 2.5 & & & 0.18888 & 0.16034 & 0.17315 & 0.17903 & 0.18128 & 0.01967 & 0.00961 & 0.00611 & 0.00459 \\
\hline & 3.5 & & & 0.09697 & 0.07736 & 0.08594 & 0.09 & 0.09158 & 0.01751 & 0.00910 & 0.00588 & 0.00449 \\
\hline \multirow[t]{8}{*}{2.5} & 0.5 & 1 & & 0.81873 & 0.81273 & \begin{tabular}{|l}
0.81559 \\
\end{tabular} & 0.81708 & 0.81702 & 0.00118 & 0.00063 & 0.00038 & 0.00030 \\
\hline & 1.5 & & & 0.54881 & 0.53677 & \begin{tabular}{|l}
0.54261 \\
\end{tabular} & 0.54552 & 0.54542 & 0.00683 & 0.00370 & 0.00227 & 0.00180 \\
\hline & 2.5 & & & 0.36788 & 0.35445 & 0.36109 & 0.36423 & 0.36414 & 0.01226 & 0.00673 & 0.00417 & 0.00331 \\
\hline & 3.5 & & & 0.2466 & 0.23403 & 0.24035 & 0.24319 & 0.24314 & 0.0156 & 0.00867 & 0.00541 & 0.00430 \\
\hline & 0.5 & 3 & & 0.81873 & 0.80162 & 0.81007 & 0.81343 & 0.81427 & 0.00161 & 0.00074 & 0.00042 & 0.00033 \\
\hline & 1.5 & & & 0.54881 & 0.51519 & 0.53173 & 0.53825 & 0.53995 & 0.00892 & 0.00425 & 0.00249 & 0.00194 \\
\hline & 2.5 & & & 0.36788 & 0.33118 & 0,34915 & 0.35619 & 0.35809 & 0.01529 & 0.00754 & 0.00450 & 0.00353 \\
\hline & 3.5 & & & 0.2466 & 0.21295 & 0.22934 & 0.23573 & 0.23751 & 0.01859 & 0.00947 & 0.00574 & 0.00453 \\
\hline
\end{tabular}




\section{Comparison Bayes Estimators of Reliability in the Exponential Distribution}

\section{Discussion}

In general, as we see in the tables (4-1to 4-8) by using different estimation methods, we find the Mean Square Errors (MSE) and Mean weighted squared Errors (MWSE) were decreased when sample size increased in all cases. That means the estimation of $(R(t))$ get better for the large sample sizes. We obtained a good estimation according to the smallest values of MSE for all samples sizes (n) comparative to the other estimated values for MSE.

As we see in table (4-1), when the true value of $\theta(\theta=0.5)$ and the prior distribution for $\theta$ is

- Inverse Chi-square distribution with $v=4$ for $t=0.5$ and with $v=6$ for $t=1.5,2.5$

,3.5.

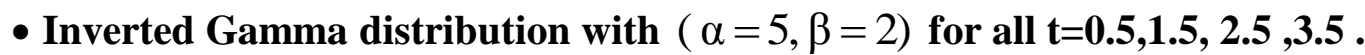

- Improper distribution with $(a=7, b=3)$ for $t=0.5$ and with $(a=9, b=3)$ for $\mathrm{t}=1.5,2.5,3.5$.

- Non-informative distribution with $\mathrm{c}=1$ for $\mathrm{t}=0.5$ and with $\mathrm{c}=3$ for $\mathrm{t}=1.5,2.5,3.5$. And in table (4-2), when the true value of $\theta(\theta=1)$ and the prior distribution for $\theta$ is

- Inverse Chi-square distribution with $v=4$ for all $t$.

- Inverted Gamma distribution with $(\alpha=3, \beta=1)$ for $\mathbf{t = 0 . 5 , 1 . 5}$ and with $(\alpha=5, \beta=2)$ for $\mathbf{t}=\mathbf{2 . 5}, \mathbf{3 . 5}$.

- Improper distribution with $(a=7, b=3)$ for all $t$.

- Non-informative distribution with $\mathrm{c}=1$ for $\mathrm{t}=0.5$ and with $\mathrm{c}=3$ for $\mathrm{t}=1.5,2.5,3.5$. in table (4-3), when the true value of $\theta(\theta=1.5)$ and the prior distribution for $\theta$ is

- Inverse Chi-square distribution with $v=4$ for $t=0.5$ and with $v=6$ for $t=1.5,2.5$ ,3.5.

- Inverted Gamma distribution with $(\alpha=3, \beta=1)$ for $\mathbf{t}=\mathbf{0 . 5 , 1 . 5 , 2 . 5}$ and with $(\alpha=5, \beta=2)$ for $t=3.5$.

- Improper distribution with $(a=7, b=3)$ for all $t$.

- Non-informative distribution with $\mathrm{c}=1$ for $\mathrm{t}=\mathbf{0 . 5 , 1 . 5}$ and with $\mathrm{c}=3$ for $\mathrm{t}=2.5,3.5$. in table (4-4), when the true value of $\theta(\theta=2.5)$ and the prior distribution for $\theta$ is

- Inverse Chi-square distribution with $\mathrm{v}=4$ for all $\mathrm{t}$.

- Inverted Gamma distribution with $(\alpha=3, \beta=1)$ for all $t$.

- Improper distribution with $(a=7, b=3)$ for all $t$.

- Non-informative distribution with $c=1$ for for all $t$. 


\section{Comparison Bayes Estimators of Reliability in the Exponential Distribution}

See the summarized and tabulated the above discussion in table (5-1) in Appendix-E..Also, we obtained a good estimation according to the smallest values of MWSE for all samples sizes (n) comparative to the other estimated values for MWSE.

In table (4-5), when the true value of $\theta(\theta=0.5)$ and the prior distribution for $\theta$ is

- Inverse Chi-square distribution with $v=4$ for $t=0.5,1.5$ and with $v=6$ for $t=2.5$ ,3.5.

- Inverted Gamma distribution with $(\alpha=5, \beta=2)$ for all $t=0.5,1.5,2.5,3.5$.

- Improper distribution with $(a=7, b=3)$ for $t=0.5,1.5$ and with $(a=9, b=3)$ for $\mathbf{t}=2.5,3.5$.

- Non-informative distribution with $\mathrm{c}=1$ for $\mathrm{t}=0.5$ and with $\mathrm{c}=3$ for $\mathrm{t}=1.5,2.5,3.5$.

In table (4-6), when the true value of $\theta(\theta=1)$ and the prior distribution for $\theta$ is

- Inverse Chi-square distribution with $v=4$ for all $t$.

- Inverted Gamma distribution with $(\alpha=3, \beta=1)$ for all $t$.

- Improper distribution with $(a=7, b=3)$ for all $t$.

- Non-informative distribution with $\mathrm{c}=1$ for $\mathrm{t}=0.5,1.5$ and with $\mathrm{c}=3$ for $\mathrm{t}=2.5,3.5$. In table (4-7) and table (4-8), when the true value of $\theta(\theta=1.5), \theta=2.5$ and the prior distribution for $\theta$ is

- Inverse Chi-square distribution with $v=4$ for all $t$.

- Inverted Gamma distribution with $(\alpha=3, \beta=1)$ for all t.

- Improper distribution with $(a=7, b=3)$ for all $t$.

- Non-informative distribution with $c=1$ for all $t$.

See the summarized and tabulated the above discussion in table (5-2) in Appendix-E.

\section{Conclusion}

When we compared the estimated values for Reliability( $R(t))$ of the Exponential distribution by using Bayes with respect to Mean Square Errors (MSE) and Mean weighted squared Errors (MWSE) in this study. We find that MSE and MWSE were decreased when sample size increased in all cases. The best method is the bayes estimation according to the smallest values of MSE for all sample sizes (n), when the prior distribution for $\theta$ is

- Improper distribution with $(a=7, b=3)$ for $t=0.5$ and with $(a=9, b=3)$ for $t=1.5$, 2.5, 3.5, when the true value of $\theta(\theta=0.5)$.

- Non-informative distribution with $\mathrm{c}=1$ for $\mathrm{t}=\mathbf{0 . 5}$, inverted Gamma distribution with $(\alpha=3, \beta=1)$ for $t=1.5$ and improper distribution with $(a=7, b=3)$ for $t=2.5$, 3.5, when the true value of $\theta(\theta=1)$.

- Non-informative distribution with $c=1$ for $t=0.5,1.5$ and inverse Chi-square distribution with $v=4$ for $t=2.5$, and inverted Gamma distribution with $(\alpha=5, \beta=2)$ for $t=1.5$, when the true value of $\theta(\theta=1.5)$. 


\section{Comparison Bayes Estimators of Reliability in the Exponential Distribution}

- Non-informative distribution with $c=1$ for all $t$, when the true value of $\theta(\theta=2.5)$.

See the the summary of conclusion for $\operatorname{MSE}(\hat{R}(t))$ in table (6-1) in Appendix-E. The best method is the bayes estimation according to the smallest values of MWSE for all samples sizes (n) when the prior distribution is

- Improper distribution with $(a=7, b=3)$ for $t=0.5,1.5$ and with $(a=9, b=3)$ for $t=$ 2.5, 3.5 when the true value of $\theta(\theta=0.5)$.

- Inverted Gamma distribution with $(\alpha=3, \beta=1)$ for $\mathbf{t}=\mathbf{0 . 5}, 1.5$ and inverse Chi-square distribution with $\mathbf{v}=4$ for $\mathbf{t}=\mathbf{2 . 5 , 3 . 5}$, when the true value of $\theta(\theta=1)$.

- Non-informative distribution with $c=1$ for all $t$, when the true value of $\theta(\theta=1.5 \& \theta=2.5)$.

See the the summary of conclusion for $\operatorname{MWSE}(R(t))$ in table (6-2) in Appendix-E.

\section{References:}

1.Baklizi, A., (2003), 'Shrinkage Estimation of the Exponential Reliability with censored data'. Focus on Applied Statistics, Nova Science Publishers Inc. Ed. Mhd. Ahsanullah. 195-204.

2.Balakrishnan, N., Lin, C.T. And. Chan, P.S, (2005),' A comparison of two simple prediction intervals for exponential distribution'. IEEE Trans. Reliab., 54(01): 27-33.

3.Bickel. P.J. \& Doksum, K. A., (1977). Mathematical Statistics: Basic Ideas and Selected Topics ", Holden- Day, Inc., San Francisco.

4.Chiou, P. (1993),'Empirical Bayes shrinkage estimation of reliability in the exponential distribution'.comm. statistic, 22(5):1483-1494.

5.Friesl,M., and Hurt ,J.(2007), ' On Bayesian estimation in exponential distribution under random censorship ', Kybernetika -Vol. (4 3) ,No.(1), Pages( $45-60)$

6.Liu, M and Ren, H., (2013),'Empirical Bayes Estimation for Exponential Model Using Non-parameter Polynomial Density Estimator', Research Journal of Applied Sciences, Engineering and Technology 5(2): 392-397.ISSN: 2040-7459; E-ISSN: 2040-7467. (C) Maxwell Scientific Organization, 2013.

7.Sarhan, A. M., (2003),' Empirical Bayes estimates in exponential reliability model'. Appl. Math. Comput. 135, 2-3, 319-332.

8.The inverse gamma distribution. (2015). Available at: From Wikipedia, the free encyclopedia. This page was last modified on 30 October 2015, http://en.wikipedia.org/wiki.

The inverse-chi-squared distribution. (2013). Available at: From Wikipedia, the free encyclopedia. This page was last modified on 19 April 2014, http://en.wikipedia.org/wiki 


\section{Comparison Bayes Estimators of Reliability in the Exponential Distribution}

\section{Appendix-A: The posterior distribution using different Priors.}

1. The posterior distribution using Inverse Chi-square distribution as prior:

It is asaumed that $\theta$ follows the Inverse Chi-square distribution with pdf as given below:

$P(\theta) \propto \frac{1}{2^{\frac{\gamma}{2}}} \theta^{-\frac{y}{2}-1} \exp \left(-\frac{1}{2 \theta}\right)$ for $v, \theta>0$

Then the posterior distribution of given the data $t=\left(t_{1}, t_{2}, \ldots, t_{n}\right)$ is:

$$
P(\theta \backslash t)-\frac{L(t \backslash \theta) P(\theta)}{\int_{\theta}^{L}(\underline{t} \backslash \theta) P(\theta) d \theta}
$$

Substituting the equation (4) and the equation (A.1) in equation (A.2), we get:

$$
\begin{aligned}
& \mathrm{P}_{1}(\theta \backslash t)-\frac{\theta^{-\mathrm{n}} \exp \left(-\frac{\sum_{\mathrm{i}-1}^{\mathrm{n}} \mathrm{t}_{\mathrm{i}}}{\theta}\right)\left[\frac{1}{2^{\frac{v}{2}}} \theta^{-\frac{\mathrm{v}}{2}-1} \exp \left(-\frac{1}{2 \theta}\right)\right]}{\int_{0}^{\infty} \theta^{-\mathrm{n}} \exp \left(-\frac{\sum_{\mathrm{i}-1}^{\mathrm{n}} \mathrm{t}_{\mathrm{i}}}{\theta}\right)\left[\frac{1}{2^{\frac{\gamma}{2}}} \theta^{-\frac{\mathrm{v}}{2}-1} \exp \left(-\frac{1}{2 \theta}\right)\right] d \theta} \\
& P_{1}(\theta \backslash t)-\frac{e^{-\left[\left(n+\frac{v}{2}\right)+1\right]} e^{-\frac{1}{\theta}\left(\sum_{i=1}^{n} t_{i}+\frac{1}{2}\right)}}{\int_{0}^{\infty} e^{-\left[\left(n+\frac{v}{2}\right)+1\right]} e^{-\frac{1}{\theta}\left(\sum_{i-1}^{n} t_{i}+\frac{1}{2}\right)} d \theta}
\end{aligned}
$$

By multiplying the integral in equation (A.4) by the quantity which equals to

$\left(\frac{\left(\sum_{\mathrm{i}-1}^{\mathrm{n}} \mathrm{t}_{\mathrm{i}}+\frac{1}{2}\right)^{\left(\mathrm{n}+\frac{2}{2}\right)}}{\Gamma\left(\mathrm{n}+\frac{v}{2}\right)}\right)\left(\frac{\Gamma\left(\mathrm{n}+\frac{v}{2}\right)}{\left(\sum_{\mathrm{i}-1}^{\mathrm{n}} \mathrm{t}_{\mathrm{i}}+\frac{1}{2}\right)^{\left(\mathrm{n}+\frac{2}{2}\right)}}\right)$, where $\Gamma($.$) is a gamma function. Then we get,$

$P_{1}(\theta \backslash t)-\frac{\left(\sum_{i=1}^{n} t_{i}+\frac{1}{2}\right)^{\left(n+\frac{y}{2}\right)} \theta^{-\left[\left(n-\frac{v}{2}\right)+1\right]} \exp \left(-\frac{1}{\theta}\left(\sum_{i=1}^{n} t_{i}+\frac{1}{2}\right)\right)}{\Gamma\left(n+\frac{v}{2}\right) A(t ; \theta)}$

Where $A(t \oplus)$ equals to

$A(t ; \theta)-\int_{0}^{\infty} \frac{\left(\sum_{i-1}^{n} t_{i}+\frac{1}{2}\right)^{\left(n+\frac{v}{2}\right)} \theta^{-\left[\left(n+\frac{v}{2}\right)+1\right]} \exp \left(-\frac{1}{\theta}\left(\sum_{i-1}^{n} t_{i}+\frac{1}{2}\right)\right)}{\Gamma\left(n+\frac{v}{2}\right)} d \theta-1$. Be the integral of the pdf of the Inverted Gamma distribution. Then we get the posterior distribution of $\theta$ given the data $\underline{t}=\left(t_{1}, t_{2}, \ldots, t_{n}\right)$ is 


\section{Comparison Bayes Estimators of Reliability in the Exponential Distribution}

$$
\begin{aligned}
& P_{1}(\theta \backslash t)-\frac{\left(\sum_{i-1}^{n} t_{i}+\frac{1}{2}\right)^{\left(n+\frac{N}{2}\right)}}{\Gamma\left(n+\frac{v}{2}\right)} \theta^{-\left[\left(n+\frac{\mathrm{v}}{2}\right)+1\right]} \exp \left(-\frac{1}{\theta}\left(\sum_{i=1}^{\mathrm{n}} t_{1}+\frac{1}{2}\right)\right) \\
& \text { It means that } \left.P_{1}(\theta \backslash t)\right) \sim \text { Inverted Gamma distribution with new }
\end{aligned}
$$

2. The posterior distribution using Inverted Gamma distribution as prior:

It is assumed that $\theta$ follows the Inverted Gamma distribution with pdf as given below:

$P(\theta) \propto \frac{\beta^{\alpha}}{\Gamma \alpha} \theta^{-(\alpha+1)} \exp \left(-\frac{\beta}{\theta}\right) \quad$ for $\alpha, \beta, \theta>0$

Then the posterior distribution of given the data $t=\left(t_{1}, t_{2}, \ldots, t_{n}\right)$ according to the equation (A.2), we get it by aubstituting the equation (4) and the equation (A.7) in equation (A.2), so we have

$P_{2}(\theta \backslash t)-\frac{\theta^{-\mathrm{n}} \exp \left(-\frac{\sum_{\mathrm{i}-1}^{\mathrm{n}}{ }_{\mathrm{t}}}{\theta}\right)\left[\frac{\beta^{\alpha}}{\Gamma \alpha} \theta^{-(\alpha+1)}\right.}{\int_{0}^{\infty} \theta^{-\mathrm{n}} \exp \left(-\frac{\sum_{\mathrm{i}-1}^{\mathrm{n}}{ }_{\mathrm{i}}}{\theta}\right)\left[\frac{\beta^{\alpha}}{\Gamma \alpha} \theta^{-(\alpha+1)} \exp \left(-\frac{\beta}{\theta}\right)\right]}$

$P_{2}(\theta \backslash t)-\frac{\theta^{-[(n+\alpha)+1]} \exp \left(-\frac{1}{\theta}\left(\Sigma_{i-1}^{n} t_{i}+\beta\right)\right)}{\int_{0}^{\infty} \theta^{-[(n+\alpha)+1]} \exp \left(-\frac{1}{\theta}\left(\Sigma_{i-1}^{n} t_{i}+\beta\right) d \theta\right.}$

By multiplying the integral in equation (A.9) by the quantity which equals to

$\left(\frac{\left(\sum_{i=1}^{n} t_{i}+\beta\right)^{(n+\infty)}}{\Gamma(n+\alpha)}\right)\left(\frac{\Gamma(n+\alpha)}{\left(\sum_{i=1}^{n} t_{i}+\beta\right)^{(n+\alpha)}}\right)$, where $\Gamma($.$) is a gamma function. Then we get,$

$P_{2}(\theta \backslash t)-\frac{\left(\Sigma_{i=1}^{n} t_{i}+\beta\right)^{(n+\alpha)} \theta^{-[(n+\alpha)+1]} \exp \left(-\frac{1}{\theta}\left(\Sigma_{i-1}^{n} t_{i}+\beta\right)\right.}{\Gamma(n+\alpha) B(t ; \theta)}$

Where $B(t \oplus)$ equals to

$P_{2}(\theta \backslash t)-\frac{\left(\sum_{i=1}^{n} t_{i}+\beta\right)^{(n+\alpha)}}{\Gamma(n+\alpha)} \theta^{-(n)+\alpha)+1]} \exp \left(-\frac{1}{\theta}\left(\sum_{i-1}^{n} t_{1}+\beta\right)\right)$

$B(t ; \theta)-\int_{0}^{\infty} \frac{\left.\left(\sum_{i=1}^{n} t_{i}+\beta\right)^{(n+\alpha)}\right)}{\Gamma(n+\alpha)} \theta^{-f(n+\infty)+\infty)+1]} \exp \left(-\frac{1}{\theta}\left(\sum_{i-1}^{n} t_{1}+\beta\right) d \theta-1\right.$. Be the integral of the pdf of the Inverted Gamma distribution. Then we get the posterior distribution of $\theta$ given the data $\underline{t}=\left(t_{1}, t_{2}, \ldots, t_{n}\right)$ is 


\section{Comparison Bayes Estimators of Reliability in the Exponential Distribution}

It means that $P_{2}(\theta \backslash t) \sim$ Inverted Gamma distribution with new

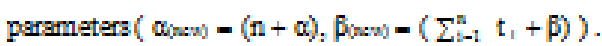

3. The posterior distribution using improper distribution as prior:

It is assumed that $\theta$ follows the improper distribution with pdf as given below:

$P(\theta) a \theta^{-(a+1)} \exp \left(-\frac{b}{\theta}\right) \quad$ for $b, \theta>0$ and $-\infty<a<\infty$

Then the posterior distribution of given the data $t=\left(t_{1}, t_{2}, \ldots, t_{n}\right)$ according to the equation (A.2), we get it by substituting the equation (4) and the equation (A.12) in equation (A.2), so we have

$$
\begin{gathered}
P_{2}(\theta \backslash t)-\frac{\theta^{-n} \exp \left(-\frac{\sum_{i=1}^{n} t_{i}}{\theta}\right)\left[\theta^{-(a+1)} \exp \left(-\frac{b}{\theta}\right)\right]}{\int_{0}^{\infty} \theta^{-n} \exp \left(-\frac{\sum_{i=1}^{n}{ }^{t}}{\theta}\right)\left[\theta^{-(a+1)} \exp \left(-\frac{b}{\theta}\right)\right] d \theta} \\
P_{2}(\theta \backslash t)-\frac{\theta^{-[(n+a)+1]} \exp \left(-\frac{1}{\theta}\left(\sum_{i=1}^{n} t_{i}+b\right)\right)}{\int_{0}^{\infty} \theta^{-[(n+a)+1]} \exp \left(-\frac{1}{\theta}\left(\sum_{i=1}^{n} t_{i}+b\right)\right) d \theta}
\end{gathered}
$$

By multiplying the integral in equation (A.14) by the quantity which equals to

$$
\begin{aligned}
& \left(\frac{\left(\sum_{\mathrm{i}=1}^{\mathrm{n}} \mathrm{t}_{\mathrm{i}}+\mathrm{b}\right)^{(\mathrm{n}+\mathrm{a})}}{\Gamma(\mathrm{n}+\mathrm{a})}\right)\left(\frac{\Gamma(\mathrm{n}+\mathrm{a})}{\left(\sum_{\mathrm{i}-1}^{\mathrm{n}} \mathrm{t}_{\mathrm{i}}+\mathrm{b}\right)^{(\mathrm{n}+\mathrm{a})}}\right) \text {, where } \Gamma(.) \mathrm{is} \text { a gamma function . Then we get, } \\
& \mathrm{P}_{2}(\theta \backslash \mathrm{t})-\frac{\left(\sum_{\mathrm{i}=1}^{\mathrm{n}} \mathrm{t}_{\mathrm{i}}+\mathrm{b}\right)^{(\mathrm{a}+\mathrm{a})} \theta^{-[(\mathrm{n}+\mathrm{a})+1]} \exp \left(-\frac{1}{\theta}\left(\sum_{\mathrm{i}-1}^{\mathrm{n}} \mathrm{t}_{\mathrm{i}}+\mathrm{b}\right)\right)}{\Gamma(\mathrm{n}+\mathrm{a}) \mathrm{C}(\mathrm{t} ; \theta)}
\end{aligned}
$$

Where $C(x, \theta)$ equals to

$C(t ; \theta)-\int_{0}^{\infty} \frac{\left(\sum_{i=1}^{n} t_{i}+b\right)^{(n+a)} \theta^{-[(n+a)+1]} \exp \left(-\frac{1}{\theta}\left(\sum_{i=1}^{n} t_{i}+b\right)\right)}{\Gamma(n+a)} d \theta-1$. Be the integral of the pdf of the Inverted Gamma distribution. Then we get the posterior distribution of $\theta$ given the data $\underline{t}=\left(t_{1}, t_{2}, \ldots, t_{n}\right)$ is

$$
\begin{aligned}
& P_{2}(\theta \backslash t)-\frac{\left(\sum_{i=1}^{n} t_{i}+b\right)^{(n+a)}}{\Gamma(n+a)} \theta^{i(n+2)+1]} \exp \left(-\frac{1}{\theta}\left(\sum_{i-1}^{n} t_{1}+b\right)\right) \\
& \text { It means that } P_{2}(\theta \backslash t) \sim \text { Inverted Gamma distribution with new } \\
& \text { parameters }\left(\alpha \text { meno }-(n+a), \beta_{\text {(new })}-\left(\sum_{i=1}^{n} t_{1}+b\right)\right) \text {. }
\end{aligned}
$$




\section{Comparison Bayes Estimators of Reliability in the Exponential Distribution}

4. The posterior distribution using Non-informative distribution as prior:

It is assumed that $\theta$ follows the non-informative distribution with pdf as given below:

$\mathrm{P}(\theta) \propto \frac{1}{\theta^{e}} \quad$ for $\theta, c>0$

Then the posterior distribution of given the data $t=\left(t_{1}, t_{2}, \ldots, t_{n}\right)$ according to the equation (A.2), we get it by substituting the equation (4) and the equation (A.17) in equation (A.2), so we have

$\mathrm{P}_{4}(\theta \backslash \mathrm{t})-\frac{\theta^{-\mathrm{n}} \exp \left(-\frac{\sum_{\mathrm{i}-1}^{\mathrm{n}}{ }^{\mathrm{t}}{ }_{\mathrm{i}}}{\theta}\right)\left[\theta^{-\mathrm{c}}\right]}{\int_{0}^{\infty} \theta^{-\mathrm{n}} \exp \left(-\frac{\sum_{\mathrm{i}-1}^{\mathrm{n}}{ }_{\mathrm{i}}}{\theta}\right)\left[\theta^{-\mathrm{c}}\right] d \theta}$
$\mathrm{P}_{4}(\theta \backslash \mathrm{t})-\frac{\int_{0}^{\infty} \theta^{-(\mathrm{n}+\mathrm{n}+\mathrm{c})} \exp \left(-\frac{1}{\theta} \sum_{\mathrm{i}-1}^{\mathrm{n}} \mathrm{exp}_{\mathrm{i}}\right)}{\left.\mathrm{t}_{\mathrm{i}}-\frac{1}{\theta} \sum_{\mathrm{i}-1}^{\mathrm{n}} \mathrm{t}_{\mathrm{i}}\right) \mathrm{d \theta}}$

We can write $\theta^{-(n+c)}$ as $\theta^{-[(a+c-1)+1]}$, and by multiplying the integral in equation (A.19), by the quantity which equals to

$$
\begin{aligned}
& \left(\frac{\left(\sum_{\mathrm{i}=1}^{\mathrm{n}} \mathrm{t}_{\mathrm{i}}\right)^{(\mathrm{n}+--1)}}{\Gamma(\mathrm{n}+\mathrm{c}-1)}\right)\left(\frac{\Gamma(\mathrm{n}+\mathrm{c}-1)}{\left(\sum_{\mathrm{i}-1}^{\mathrm{n}} \mathrm{t}_{\mathrm{i}}\right)^{(\mathrm{n}+--1)}}\right) \text {, where } \Gamma(.) \text { is a gamma function. Then we get } \\
& \mathrm{P}_{4}(\theta \backslash \mathrm{t})-\frac{\left(\sum_{\mathrm{i}=1}^{\mathrm{n}} \mathrm{t}_{\mathrm{i}}\right)^{(\mathrm{n}+c-1)} \theta^{-[(\mathrm{n}+c-1)+1]} \exp \left(-\frac{1}{\theta} \Sigma_{\mathrm{i}-1}^{\mathrm{n}} \mathrm{t}_{\mathrm{i}}\right)}{\Gamma(\mathrm{n}+\mathrm{c}-1) \mathrm{D}(\mathrm{t} ; \theta)}
\end{aligned}
$$

Where $D(x, \theta)$ equals to

$$
\mathrm{D}(\mathrm{t} ; \theta)=\int_{0}^{\infty} \frac{\left(\sum_{\mathrm{i}-1}^{\mathrm{n}} \mathrm{t}_{\mathrm{i}}\right)^{(\mathrm{n}+\mathrm{c}-1)} \theta^{-[(\mathrm{n}+\mathrm{c}-1)+1]} \exp \left(-\frac{1}{\theta} \Sigma_{\mathrm{i}-1}^{\mathrm{n}} \mathrm{t}_{\mathrm{i}}\right)}{\Gamma(\mathrm{n}+\mathrm{c}-1)} d \theta-1 \text {. Be the integral of the }
$$

pdf of the Inverted Gamma distribution. Then we get the posterior distribution of $\theta$ given the data $\underline{t}=\left(\mathrm{t}_{1}, \mathrm{t}_{2}, \ldots, \mathrm{t}_{\mathrm{a}}\right)$ is

$P_{s}(\theta \backslash t)-\frac{\left(\Sigma_{i=1}^{n} t_{i}\right)^{(n+c-1)}}{\Gamma(n+c-1)} \theta^{-j i n-c-1)+1]} \exp \left(-\frac{1}{\theta} \sum_{i=1}^{n} t_{1}\right)$

It means that $P_{4}(\theta \backslash t)$ - Inverted Gamma distribution with new

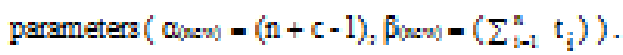




\section{Comparison Bayes Estimators of Reliability in the Exponential Distribution}

Appendix-B: The following is the derivation of these estimators under the squared error loss function.

1. The squared error loss function

To obtain the Bayes' estimator, we minimize the posterior expected loss given by:

$L_{1}(R, R)-(R-R)^{2}$, where $R-R(t) \& R-R(t)$, the risk function is:

Risk - $\mathrm{E}\left[\mathrm{L}_{1}(\mathrm{R}, \mathrm{R})\right]$

Risk - E[( R-R $\left.)^{2}\right]$

Risk $-\int_{\theta}(\hat{R}-R)^{2} P(\theta \backslash t) d \theta$

Risk $-\int_{\theta}(\hat{R}-R)^{2} P(\theta \backslash t) d \theta \Rightarrow R i s k-\int_{\theta}\left(\hat{R}^{2}-2 R \hat{R}+R^{2}\right) P(\theta \backslash t) d \theta$

Risk $-\hat{R}^{2} \int_{0}^{\pi} P(\theta \backslash t) d \theta-2 \hat{R} \int_{0}^{\pi} R P(\theta \backslash t) d \theta+\int_{0}^{\pi} R^{2} P(\theta \backslash t) d \theta \Rightarrow$

Risk $=\hat{R}^{2}-2 \hat{R} E(R \backslash t)+E\left(R^{2} \backslash t\right)$

Let $\frac{\partial}{\hat{n}}$ Risk -0 , we get Bayes estimator of $R$ denoted by $\hat{R}_{\text {ayea }}$ for the above prior as follows

$\hat{R}_{s=}(t)-E(R \backslash t)-\int_{0}^{\pi} R(t) P(\theta \backslash t) d \theta$

$\hat{\mathbf{R}}_{\mathrm{sz}}(\mathrm{t})-\int_{0}^{\pi} \mathrm{R}(\mathrm{t}) \mathrm{P}_{2}(\theta \backslash \mathrm{t}) \mathrm{d \theta} \quad, \mathrm{i}-1,2,3,4$

1.1 Baves estimation using Inverse chi-squared distribution as prior.

To obtain the Bayes' estimator under inverse chi-squared distribution as prior. Substituting the equation (A. 6) in equation (B.4), we get:

$\hat{R}_{m=1}(t)-\int_{0}^{0} R(t) P_{1}(\theta \backslash t) d \theta \quad$ for $\quad i-1$

$\hat{R}_{n=1}(t)-\int_{0}^{e} \exp \left(-\frac{t}{\theta}\right) \frac{\left(\Sigma_{i-1}^{n} t_{i}+\frac{1}{2}\right)^{\left(n+\frac{y}{2}\right)}}{\Gamma\left(n+\frac{v}{2}\right)} \theta^{-\left[\left(n+\frac{y}{2}\right)+1\right]} \exp \left(-\frac{1}{\theta}\left(\sum_{i=1}^{\mathrm{n}} t_{1}+\frac{1}{2}\right)\right) d \theta$

$\hat{R}_{n=1}(t)-\int_{0}^{\infty} \frac{\left(\sum_{i=1}^{n} t_{i}+\frac{1}{2}\right)^{\left(n+\frac{2}{2}\right)}}{\Gamma\left(n+\frac{v}{2}\right)} \theta^{-\left[\left(n+\frac{v}{2}\right)+1\right]} \exp \left(-\frac{1}{\theta}\left(\sum_{i=1}^{n} t_{1}+t+\frac{1}{2}\right)\right) d \theta$

By multiplying the integral in equation (B.6) by the quantity which equals to $x_{i}-\frac{\left(\Sigma_{i-1}^{n} t_{i}+t+\frac{1}{2}\right)^{\left(n+\frac{v}{2}\right)}}{\left(\Sigma_{i-1}^{n} t_{i}+t+\frac{1}{2}\right)^{\left(n+\frac{v}{2}\right)}}$, so we have 


\section{Comparison Bayes Estimators of Reliability in the Exponential Distribution}

$\hat{R}_{n=1}(t)-x_{i} \int_{0}^{\infty} \frac{\left(\sum_{i-1}^{n} t_{i}+\frac{1}{2}\right)^{\left(n+\frac{3}{2}\right)}}{\Gamma\left(n+\frac{v}{2}\right)} \theta^{-\left[\left(n+\frac{W}{2}\right)+1\right]} \exp \left(-\frac{1}{\theta}\left(\sum_{i-1}^{n} t_{1}+t+\frac{1}{2}\right)\right) d \theta$

Then we have

$\hat{R}_{n=1}(t)-\frac{\left(\sum_{i=1}^{n} t_{i}+\frac{1}{2}\right)^{\left(n+\frac{y}{2}\right)}}{\left(\sum_{i=1}^{n} t_{i}+t+\frac{1}{2}\right)^{\left(n+\frac{y}{2}\right)}}\left(x_{2}(t ; \theta)\right)$

Where $x_{2}(t, \theta)$ equals to

$x_{2}(t ; \theta)-\int_{0}^{0} \frac{\left(\sum_{i-1}^{n} t_{i}+t+\frac{1}{2}\right)^{\left(n+\frac{y}{2}\right)}}{\Gamma\left(n+\frac{v}{2}\right)} e^{-\left[\left(n+\frac{v}{2}\right)+1\right]} \exp \left(-\frac{1}{\theta}\left(\left(\sum_{i=1}^{n} t_{1}+t+\frac{1}{2}\right)\right) d \theta-1\right.$. Be the integral of the pdf of the Inverted Gamma distribution. Then we get the Bsyes estimator of $R$ as the following formula:

$\hat{R}_{n=1}(t)-\frac{\left(\sum_{i=1}^{n} t_{i}+\frac{1}{2}\right)^{\left(n+\frac{y}{2}\right)}}{\left(\sum_{i-1}^{n} t_{i}+t+\frac{1}{2}\right)^{\left(n+\frac{y}{2}\right)}}-\left[\frac{\sum_{i=1}^{n} t_{i}+0.5}{\sum_{i=1}^{n} t_{i}+t+0.5}\right]^{\left(n+\frac{\pi}{2}\right)} \quad, n \& v>0$

1.2 Bavs estimation using Inverted gamma distribution as prior:

To obtain the Bayes' estimator under the inverted gamma distribution as prior. Substituting the equation (A.11) in equation (B.4), we get:

$$
\begin{aligned}
& \hat{R}_{2 \pi z}(t)-\int_{0}^{\pi} R(t) P_{2}(\theta \backslash t) d \theta \quad \text { for } \quad i=2 \\
& \hat{R}_{m=2}(t)-\int_{0}^{\infty} \exp \left(-\frac{t}{\theta}\right) \frac{\left(\sum_{i=1}^{n} t_{i}+\beta\right)^{(n+\alpha)}}{\Gamma(n+\alpha)} \theta^{i(j)+\infty j+1]} \exp \left(-\frac{1}{\theta}\left(\sum_{i=1}^{n} t_{1}+\beta\right)\right) d \theta \\
& \hat{R}_{n=2}(t)-\int_{0}^{0} \frac{\left(\sum_{i=1}^{n} t_{i}+\beta\right)^{(n+\alpha)}}{\Gamma(n+\alpha)} \theta^{-[(n)-\alpha)+1]} \exp \left(-\frac{1}{\theta}\left(\sum_{t=1}^{n} t_{1}+t+\beta\right)\right) d \theta \\
& \text { By multiplying the integral in equation (B.11) by the quantity which equals } \\
& \text { to } h_{1}-\frac{\left(\sum_{i-1}^{n} t_{i}+t+\beta\right)^{(n+\alpha)}}{\left(\Sigma_{i-1}^{n} t_{i}+t+\beta\right)^{(n+\alpha)}} \text {, so we have } \\
& \hat{R}_{n=2}(t)-h_{1} \int_{0}^{\infty} \frac{\left(\sum_{i-1}^{n} t_{i}+\beta\right)^{(n+\alpha)}}{\Gamma(n+\alpha)} \theta^{-j / n-\alpha)+1]} \exp \left(-\frac{1}{\theta}\left(\sum_{t=1}^{n} t_{1}+t+\beta\right)\right) d \theta \\
& \text { Then we have } \\
& \hat{R}_{m=2}(t)-\frac{\left(\sum_{i=1}^{n} t_{i}+\beta\right)^{(n+\alpha)}}{\left(\sum_{i=1}^{n} t_{i}+t+\beta\right)^{(n+\alpha)}}\left(h_{2}(t ; \theta)\right) \\
& \text { Where } h(t ; \theta) \text { equals to }
\end{aligned}
$$




\section{Comparison Bayes Estimators of Reliability in the Exponential Distribution}

$h(t ; \theta)-i \int_{0}^{\infty} \frac{\left(\Sigma_{i}^{n}-1 t_{i}+t+\beta\right)^{(n+\alpha)}}{\Gamma(n+\alpha)} \theta^{-i n+\infty)+1]} \exp \left(-\frac{1}{\theta}\left(\sum_{t-1}^{n} t_{1}+t+\beta\right)\right) d \theta-1$. Be the integral of the pdf of the Inverted Gamma distribution. Then we get the Bayes estimator of $R$ as the following formula:

$\hat{R}_{n=2}(t)-\frac{\left(\sum_{i=1}^{n} t_{i}+\beta\right)^{(n+\alpha)}}{\left(\sum_{i=1}^{n} t_{i}+t+\beta\right)^{(n+\alpha)}}-\left[\frac{\sum_{i=1}^{n} t_{i}+\beta}{\sum_{i=1}^{n} t_{i}+t+\beta}\right]^{(n+\infty)} \quad, n, \beta, \alpha>0$

1.3 Baves estimation using improper distribution as prior:

To obtain the Bayes' estimator under improper distribution as prior. Substituting the equation (A.16) in equation (B.4), we get:

$\hat{R}_{\max z}(t)-\int_{0}^{\pi} R(t) P_{2}(\theta \backslash t) d \theta$ for $\quad i-3$

$\hat{R}_{n=3}(t)-\int_{0}^{e} \exp \left(-\frac{t}{\theta}\right) \frac{\left(\Sigma_{i}^{n}-1 t_{i}+b\right)^{(n+a)}}{\Gamma(n+a)} \theta^{-0 / n+1)+1]} \exp \left(-\frac{1}{\theta}\left(\sum_{t-1}^{n} t_{1}+b\right)\right) d \theta$

$\hat{R}_{x=3}(t)-\int_{0}^{0} \frac{\left(\sum_{i-1}^{n} t_{i}+b\right)^{(n+a)}}{\Gamma(n+a)} \theta^{-0(0 n+1)+1]} \exp \left(-\frac{1}{\theta}\left(\sum_{i-1}^{n} t_{1}+t+b\right)\right) d \theta$

By multiplying the integral in equation (B.13) by the quantity which equals to $E_{i}-\frac{\left(\sum_{i-1}^{n} t_{i}+t+b\right)^{(n+a)}}{\left(\sum_{i-1}^{n} t_{i}+t+b\right)^{(n+2)}}$, so we have

$\hat{R}_{m=3}(t)-E_{i} \int_{0}^{\infty} \frac{\left(\Sigma_{i=1}^{n} t_{i}+b\right)^{(n+a)}}{\Gamma(n+a)} \theta^{-(0) m-1)+1]} \exp \left(-\frac{1}{\theta}\left(\sum_{i-1}^{n} t_{1}+t+b\right)\right) d \theta$

Then we have

$\hat{R}_{m=z}(t)-\frac{\left(\sum_{i-1}^{n} t_{i}+b\right)^{(n+a)}}{\left(\sum_{i=1}^{n} t_{i}+t+b\right)^{(n+a)}}\left(E_{2}(t ; \theta)\right)$

Where $\mathrm{E} 2(t, \theta)$ equals to

$E 2(t ; \theta)-\int_{0}^{\infty} \frac{\left(\sum_{i=1}^{n} t_{i}+t+b\right)^{(n+a)}}{\Gamma(n+a)} \theta^{-0 / 0 n+1 ; 1]} \exp \left(-\frac{1}{\theta}\left(\sum_{i-1}^{n} t_{1}+t+b\right)\right) d \theta-1$. Be the integral of the pdf of the Inverted Gamma distribution. Then we get the Bayes estimator of $R$ as the following formula:

$\hat{R}_{\min }(t)-\frac{\left(\sum_{i-1}^{n} t_{i}+b\right)^{(n+a)}}{\left(\sum_{i=1}^{n} t_{i}+t+b\right)^{(n+a)}}-\left[\frac{\sum_{i-1}^{n} t_{t}+b}{\sum_{i=1}^{n} t_{i}+t+b}\right]^{(n+a)} \quad n, b, a>0$ 


\section{Comparison Bayes Estimators of Reliability in the Exponential Distribution}

1.4 Baves estimation using non-informative distribution as prior.

To obtain the Bayes' estimator under non infortmative distribution as prior. Substituting the equation (A.21) in equation (B.4), we get:

$\hat{R}_{m \pi}(t)-\int_{0}^{\pi} R(t) P_{s}(\theta \backslash t) d \theta$ for $\quad i-4$

$\hat{R}_{n \pi d}(t)-\int_{0}^{e} \exp \left(-\frac{t}{\theta}\right) \frac{\left(\sum_{i-1}^{n} t_{i}\right)^{(n+c-1)}}{\Gamma(n+c-1)} \theta^{f(n+n-1)+1]} \exp \left(-\frac{1}{\theta}\left(\sum_{i=1}^{n} t_{1}\right)\right) d \theta$

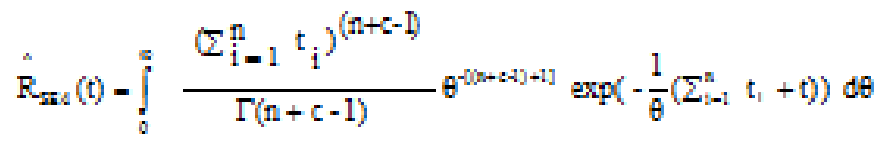

By multiplying the integral in equation (B.18) by the quantity which equals to $F_{1}=\frac{\left(\sum_{i=1}^{n} t_{i}+t\right)^{(a+c-1)}}{\left(\sum_{i=1}^{n} t_{i}+t\right)^{(n+c-1)}}$, so we have

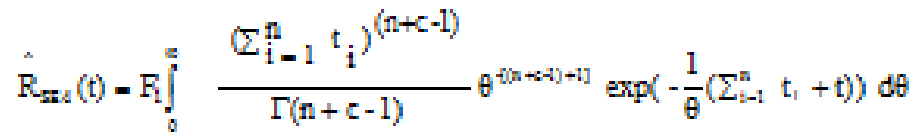

Then we have

$\hat{R}_{n i s}(t)-\frac{\left(\sum_{i=1}^{n} t_{i}\right)^{(n+c-1)}}{\left(\sum_{i=1}^{n} t_{i}+t\right)^{(n+c-1)}}\left(F_{2}(t ; \theta)\right)$

Where $F_{2}(t, \theta)$ equals to

$F(t ; \theta)-\int_{0}^{\pi} \frac{\left(\sum_{i=1}^{n} t_{i}+t\right)^{(n+c-1)}}{\Gamma(n+c-1)} \theta^{-[i n+\infty 1)+1]} \exp \left(-\frac{1}{\theta}\left(\sum_{i=1}^{n} t_{1}+t\right)\right) d \theta-1$. Bethe integral of the pdf of the Inverted Gamma distribution. Then we get the Bayes estimator of $R$ as the following formula:

$\hat{R}_{m i s}(t)-\frac{\left(\sum_{i=1}^{n} t_{i}\right)^{(n+c-1)}}{\left(\sum_{i=1}^{n} t_{i}+t\right)^{(n+c-1)}}-\left[\frac{\sum_{i=1}^{n} t_{i}}{\sum_{i=1}^{n} t_{i}+t}\right]^{(n+c-1)} \quad n, c>0$ 


\section{Comparison Bayes Estimators of Reliability in the Exponential Distribution}

Appendix-C: The following is the derivation of these estimators under the weighted squared error loss function.

2. The weighted squared error loss function

To obtain the Bayes' estimator, we minimize the posterior expected loss given by:

$L_{2}(\hat{R}, R)-K \frac{(\hat{R}-R)^{2}}{R}$, where $R-R(t) \& \hat{R}-\hat{R}(t)$, the risk function is:

Risk - $\mathrm{E}\left[\mathrm{L}_{2}(\mathrm{R}, \mathrm{R})\right]$

Risk - $E\left[K \frac{(\hat{R}-R)^{2}}{R}\right]$

Risk $-\int \frac{(\hat{R}-R)^{2}}{R} P(\theta \backslash t) d \theta$

Risk $-\int_{\theta} \mathrm{K} \frac{(\mathrm{R}-\mathrm{R})^{2}}{\mathrm{R}} \mathrm{P}(\theta \backslash \mathrm{t}) \mathrm{d \theta} \Rightarrow \mathrm{Risk}-\mathrm{K} \int \frac{1}{\mathrm{R}}\left(\mathrm{R}^{2}-2 \mathrm{R} R+\mathrm{R}^{2}\right) \mathrm{P}(\theta \backslash \mathrm{t}) \mathrm{d \theta}$

Risk $-K\left(\hat{R}^{2} \int_{0}^{\pi} \frac{1}{R} P(\theta \backslash t) d \theta-2 \hat{R} \int_{0}^{\pi} \frac{1}{R} R P(\theta \backslash t) d \theta+\int_{0}^{\pi} \frac{1}{R} R^{2} P(\theta \backslash t) d \theta \Rightarrow\right.$

Risk $-\hat{R}^{2} \int_{0}^{\pi} \frac{1}{R} P(\theta \backslash t) d \theta-2 \hat{R}(1)+E(R \backslash t)$

Let $\frac{\partial}{\hat{R}}$ Risk -0 , we get Bayes estimator of $\mathrm{R}$ denoted by $\hat{\mathrm{R}}_{\mathrm{zy} y \mathrm{a}}$ for the above prior as o $\mathrm{R}$

follows

$\hat{R}_{\max }(t)-\frac{1}{E\left(\frac{1}{R} \backslash t\right)}-\frac{1}{\int_{0}^{\infty} \frac{1}{R(t)} P(\theta \backslash x) d \theta}$

$\hat{R}_{w a x}(t)-\frac{1}{\int_{0}^{a} \frac{1}{R(t)} P_{i}(\theta \backslash t) d \theta} \quad, i-1,2,3,4$

2.1 Baves estimation using Inverse chi-squared distribution as prior:

To obtain the Bayes' estimator under inverse chi-squared distribution as prior. Substituting the equation (A.6) in the integral in equation (C.4), we get:

$E\left(\frac{1}{R} \backslash t\right)-\int_{0}^{\pi} \frac{1}{R(t)} P_{1}(\theta \backslash t) d \theta \quad$ for $\quad i=1$

$E\left(\frac{1}{R} \backslash t\right)-\int_{0}^{\infty} \exp \left(\frac{t}{\theta}\right) \frac{\left(\Sigma_{i=1}^{n} t_{i}+\frac{1}{2}\right)^{\left(n+\frac{y}{2}\right)}}{\Gamma\left(n+\frac{v}{2}\right)} \theta^{\left[\left(n+\frac{y}{2}\right)+1\right]} \exp \left(-\frac{1}{\theta}\left(\sum_{i=1}^{\mathrm{n}} t_{1}+\frac{1}{2}\right)\right) d \theta$ 


\section{Comparison Bayes Estimators of Reliability in the Exponential Distribution}

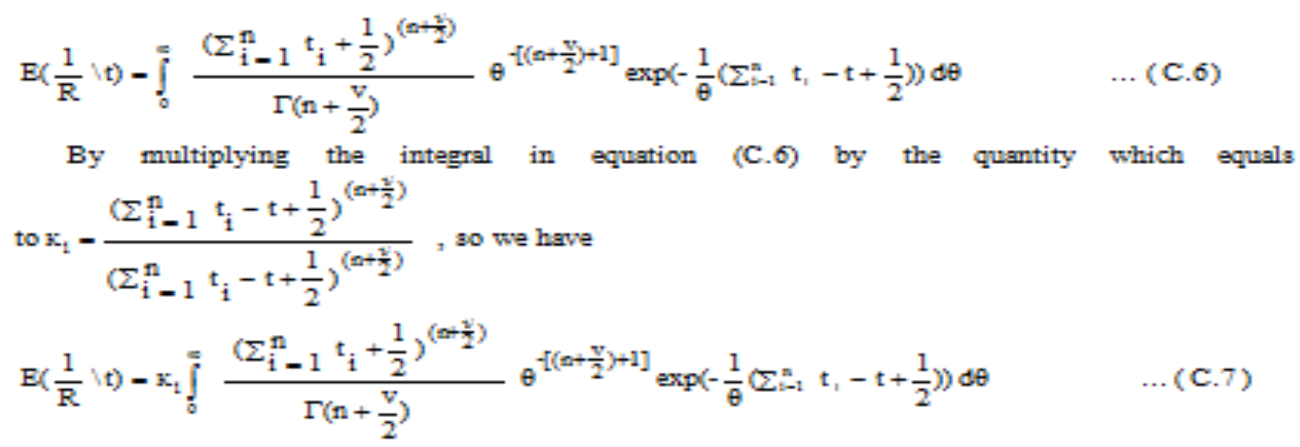

Then we have

$$
E\left(\frac{1}{R} \backslash t\right)-\frac{\left(\Sigma_{i}^{n}-1 t_{i}+\frac{1}{2}\right)^{\left(n+\frac{y}{2}\right)}}{\left(\sum_{i=1}^{n} t_{i}-t+\frac{1}{2}\right)^{\left(n+\frac{y}{2}\right)}}\left(x_{2}(t ; \theta)\right)
$$

Where $x_{2}(t, \theta)$ equals to

$\mathrm{x}_{2}(\mathrm{t} ; \theta)-\int_{0}^{=} \frac{\left(\Sigma_{\mathrm{i}-1}^{\mathrm{n}} \mathrm{t}_{\mathrm{i}}-\mathrm{t}+\frac{1}{2}\right)^{\left(\mathrm{n}+\frac{\mathrm{y}}{2}\right)}}{\Gamma\left(\mathrm{n}+\frac{\mathrm{v}}{2}\right)} \theta^{\left[\left(\mathrm{n}+\frac{\mathrm{y}}{2}\right)+1\right]} \exp \left(-\frac{1}{\theta}\left(\left(\sum_{i=1}^{\mathrm{n}} \mathrm{t}_{\mathrm{i}}-\mathrm{t}+\frac{1}{2}\right)\right) d \theta-1\right.$. Be the integral of the pdf of the Inverted Gamma distribution. So we get

$\left.E\left(\frac{1}{R}\right) t\right)-\frac{\left(\sum_{i=1}^{n} t_{i}+\frac{1}{2}\right)^{\left(n+\frac{y}{2}\right)}}{\left(\sum_{i-1}^{n} t_{i}-t+\frac{1}{2}\right)^{\left(n+\frac{y}{2}\right)}}-\left[\frac{\sum_{i-1}^{n} t_{t}+0.5}{\sum_{i=1}^{n} t_{t}-t+0.5}\right]^{\left(n+\frac{\pi}{2}\right)}$

Substituting the equation (C.9) in equation (C.4), we get the Bayes estimator of $R$ as the following formula:

$\hat{R}_{\operatorname{man} 1}(t)-\left[\frac{\sum_{t-1}^{n} t_{s}+0.5}{\sum_{t=1}^{n} t_{t}-t+0.5}\right]^{-\left(n+\frac{\pi}{2}\right)}, n \& v>0$

2.2 Bavs estimation using Inverted gamma distribution as prior:

To obtain the Bayes' estimator under the inverted gamma distribution as prior. Substituting the equation (A.11) in the integral in equation (C. 4 ), we get:

$E\left(\frac{1}{R} \backslash t\right)-\int_{0}^{\pi} \frac{1}{R(t)} P_{2}(\theta \backslash t) d \theta \quad$ for $\quad i-2$

$E\left(\frac{1}{R} \backslash t\right)-\int_{0}^{\infty} \exp \left(\frac{t}{\theta}\right) \frac{\left(\sum_{i-1}^{n} t_{i}+\beta\right)^{(n+\alpha)}}{\Gamma(n+\alpha)} \theta^{-(0)+\infty)+1]} \exp \left(-\frac{1}{\theta}\left(\sum_{i-1}^{n} t_{1}+\beta\right)\right) d \theta$

$E\left(\frac{1}{R} \backslash t\right)-\int_{0}^{\infty} \frac{\left(\sum_{i=1}^{n} t_{i}+\beta\right)^{(n+\alpha)}}{\Gamma(n+\alpha)} \theta^{-0(n+\infty)+\infty)+1]} \exp \left(-\frac{1}{\theta}\left(\sum_{t=1}^{n} t_{1}-t+\beta\right)\right) d \theta$ 


\section{Comparison Bayes Estimators of Reliability in the Exponential Distribution}

By multiplying the integral in equation (C.12) by the quantity which equals to $h_{1}-\frac{\left(\Sigma_{i-1}^{n} t_{i}-t+\beta\right)^{(n+\alpha)}}{\left(\sum_{i-1}^{n} t_{i}-t+\beta\right)^{(n+\alpha)}}$, so we have

$E\left(\frac{1}{R} \backslash t\right)-h_{1} \int_{0}^{\infty} \frac{\left(\Sigma_{i}^{n}-1 t_{i}+\beta\right)^{(n+\alpha)}}{\Gamma(n+\alpha)} \theta^{-(1 / 0)+\alpha)+1]} \exp \left(-\frac{1}{\theta}\left(\Sigma_{t-1}^{n} t_{1}-t+\beta\right)\right) d \theta$

Then we have

$E\left(\frac{1}{R} \backslash t\right)-\frac{\left(\sum_{i=1}^{n} t_{i}+\beta\right)^{(n+\alpha)}}{\left(\sum_{i=1}^{n} t_{i}-t+\beta\right)^{(n+\alpha)}}\left(h_{2}(t ; \theta)\right)$

Where $h(t, \theta)$ equals to

$h(t ; \theta)-1 \int_{0}^{\infty} \frac{\left(\sum_{i=1}^{n} t_{i}-t+\beta\right)^{(n+\alpha)}}{\Gamma(n+\alpha)} \theta^{-0(n+\infty)+1]} \exp \left(-\frac{1}{\theta}\left(\sum_{i-1}^{n} t_{1}-t+\beta\right)\right) d \theta-1$. Be the integral of the pdf of the Inverted Gamma distribution. So we get

$E\left(\frac{1}{R} \backslash t\right)-\frac{\left(\sum_{i=1}^{n} t_{i}+\beta\right)^{(n+\alpha)}}{\left(\sum_{i=1}^{n} t_{i}-t+\beta\right)^{(n+\alpha)}}-\left[\frac{\sum_{i=1}^{n} t_{i}+\beta}{\sum_{i=1}^{n} t_{i}-t+\beta}\right]^{(n+\alpha)} \quad, n, \beta, \alpha>0$

Substituting the equation (C.15) in equation (C.4), we get the Bayes estimator of $R$ as the following formula:

$\hat{R}_{w=2 \pi}(t)-\left[\frac{\sum_{i=1}^{n} t_{s}+\beta}{\sum_{i=1}^{n} t_{t}-t+\beta}\right]^{-(n+\infty)} \quad, n, \beta, \alpha>0$

2.3 Baves estimation using improper distribution as prior:

To obtain the Bayes' estimator under improper distribution as prior. Substituting the equation (A.16) in the integral in equation (C. 4 ), we get:

$E\left(\frac{1}{R} \backslash t\right)-\int_{0}^{0} \frac{1}{R(t)} P_{2}(\theta \backslash t) d \theta \quad$ for $\quad i-3$

$E\left(\frac{1}{R} \backslash t\right)-\int_{0}^{\infty} \exp \left(\frac{t}{\theta}\right) \frac{\left(\sum_{i-1}^{n} t_{i}+b\right)^{(n+a)}}{\Gamma(n+a)} \theta^{-(n+n+1)+1]} \exp \left(-\frac{1}{\theta}\left(\sum_{t-1}^{n} t_{1}+b\right)\right) d \theta$

$E\left(\frac{1}{R} \backslash t\right)-\int_{0}^{0} \frac{\left(\sum_{i}^{n}-1 t_{i}+b\right)^{(n+a)}}{\Gamma(n+a)} \theta^{-0,0 m+1 ;-1]} \exp \left(-\frac{1}{\theta}\left(\sum_{i-1}^{n} t_{1}-t+b\right)\right) d \theta$

By multiplying the integral in equation (C.18) by the quantity which equals to $E_{i}-\frac{\left(\sum_{i-1}^{n} t_{i}-t+b\right)^{(n+2)}}{\left(\Sigma_{i-1}^{n} t_{i}-t+b\right)^{(n+z)}}$, so we have 


\section{Comparison Bayes Estimators of Reliability in the Exponential Distribution}

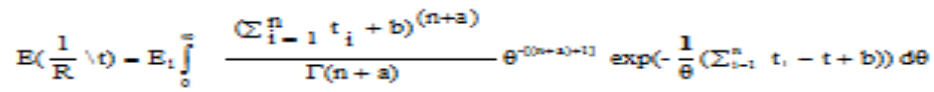

Then we have

$E\left(\frac{1}{R} \backslash t\right)-\frac{\left(\sum_{i-1}^{n} t_{i}+b\right)^{(n+a)}}{\left(\sum_{i-1}^{n} t_{i}-t+b\right)^{(n+a)}}\left(E_{2}(t ; \theta)\right)$

Where $E(t, \theta)$ equals to

$\operatorname{Ea}(t ; \theta)-\int_{0}^{\infty} \frac{\left(\Sigma_{i}^{n}-1 t_{i}-t+b\right)^{(n+a)}}{\Gamma(n+a)} \theta^{-0(0)+1) ; 1]} \exp \left(-\frac{1}{\theta}\left(\Sigma_{i-1}^{n} t_{i}-t+b\right)\right) d \theta-1$. Be the integral of the pdf of the Inverted Gamma distribution. So we get

$E\left(\frac{1}{R} \backslash t\right)-\frac{\left(\sum_{i-1}^{n} t_{i}+b\right)^{(n+a)}}{\left(\sum_{i-1}^{n} t_{i}-t+b\right)^{(n+a)}}-\left[\frac{\sum_{i=1}^{n} t_{i}+b}{\sum_{i-1}^{n} t_{i}-t+b}\right]^{(n+a)} \quad n, b, a>0 \quad \quad \ldots(C .21)$

Substituting the equation (C.21) in equation (C.4), we get the Bayes estimator of $R$ as the following formula:

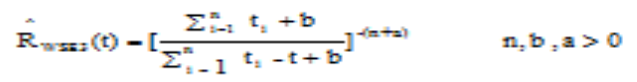

2.4 Baves estimation using non-informative distribution as prior:

To obtain the Bayes' estimator under non infortmative distribution as prior. Substituting the equation (A.21) in the integral in equation (C. 4 ), we get:

$E\left(\frac{1}{R} \backslash t\right)-\int_{0}^{\infty} \frac{1}{R(t)} P_{s}(\theta \backslash t) d \theta \quad$ for $\quad i-4$

$E\left(\frac{1}{R} \backslash t\right)-\int_{0}^{\infty} \exp \left(\frac{t}{\theta}\right) \frac{\left(\Sigma_{i}^{n}-1 t_{i}\right)^{(n+c-1)}}{\Gamma(n+c-1)} e^{-(n-m-c-1)+1]} \exp \left(-\frac{1}{\theta} \sum_{i-1}^{n} t_{1}\right) d \theta$

$E\left(\frac{1}{R} \backslash t\right)-\int_{0}^{\infty} \frac{\left(\Sigma_{i}^{n}-1 t_{i}\right)^{(n+c-1)}}{\Gamma(n+c-1)} \theta^{-[0 n+\infty)-1]-1]} \exp \left(-\frac{1}{\theta} \sum_{t-1}^{n} t,-t\right) d \theta$

By multiplying the integral in equation (C.24) by the quantity which equals to $F_{1}-\frac{\left(\sum_{i-1}^{n} t_{i}-t\right)^{(n+o-1)}}{\left(\sum_{i}^{n}-1 t_{i}-t\right)^{(n+\infty-1)}}$, so we have

$E\left(\frac{1}{R} \backslash t\right)-F_{2} \int_{0}^{\infty} \frac{\left(\Sigma_{i}^{n}-1 t_{i}\right)^{(n+c-1)}}{\Gamma(n+c-1)} e^{-[0 n+c-1)+1]} \exp \left(-\frac{1}{\theta}\left(\Sigma_{i-1}^{n} t,-t\right)\right) d \theta$

Then we have

$\hat{R}_{n=s}(t)-\frac{\left(\sum_{i-1}^{n} t_{i}\right)^{(n+c-1)}}{\left(\sum_{i-1}^{n} t_{i}-t\right)^{(n+c-1)}}\left(F_{2}(t ; \theta)\right)$

Where $F_{2}(t, \theta)$ equals to

$F(t ; \theta)-\int_{0}^{\infty} \frac{\left(\sum_{i}^{n}-1 t_{i}+t\right)^{(n+c-1)}}{\Gamma(n+c-1)} \theta^{-0 / n-c-1)+1]} \exp \left(-\frac{1}{\theta} \sum_{t-1}^{n} t_{1}+t\right) d \theta-1$. Bethe integral of the pdf of the Inverted Gamma distribution. So we get

$\left.E\left(\frac{1}{R}\right) t\right)-\frac{\left(\sum_{i-1}^{n} t_{i}\right)^{(n+c-1)}}{\left(\sum_{i=1}^{n} t_{i}-t\right)^{(n+c-1)}}-\left[\frac{\sum_{i=1}^{n} t_{i}}{\sum_{i=1}^{n} t_{i}-t}\right]^{(=+e-t)} \quad n, c>0$ Subatituting the equation (C. 27 ) in equation (C. 4 ), we get the Bayes estimator of $R$ as the
following formula:

$\hat{R}_{m=s}(t)-\left[\frac{\sum_{t-1}^{n} t_{s}}{\sum_{t-1}^{n} t_{2}-t}\right]^{-(n+c-t)} \quad n, c>0$ 


\section{Comparison Bayes Estimators of Reliability in the Exponential Distribution}

Appendix-D: The following is the programs algorithm.

Algorithm (1): To compute Bayes estimators $\left(\mathrm{R}_{\mathrm{SEl}}(\mathrm{t})\right)$ using inverse chi-square as prior distribution for $\theta$ with MSE for $\hat{\mathrm{R}}_{\mathrm{SEI}}(\mathrm{t})$.

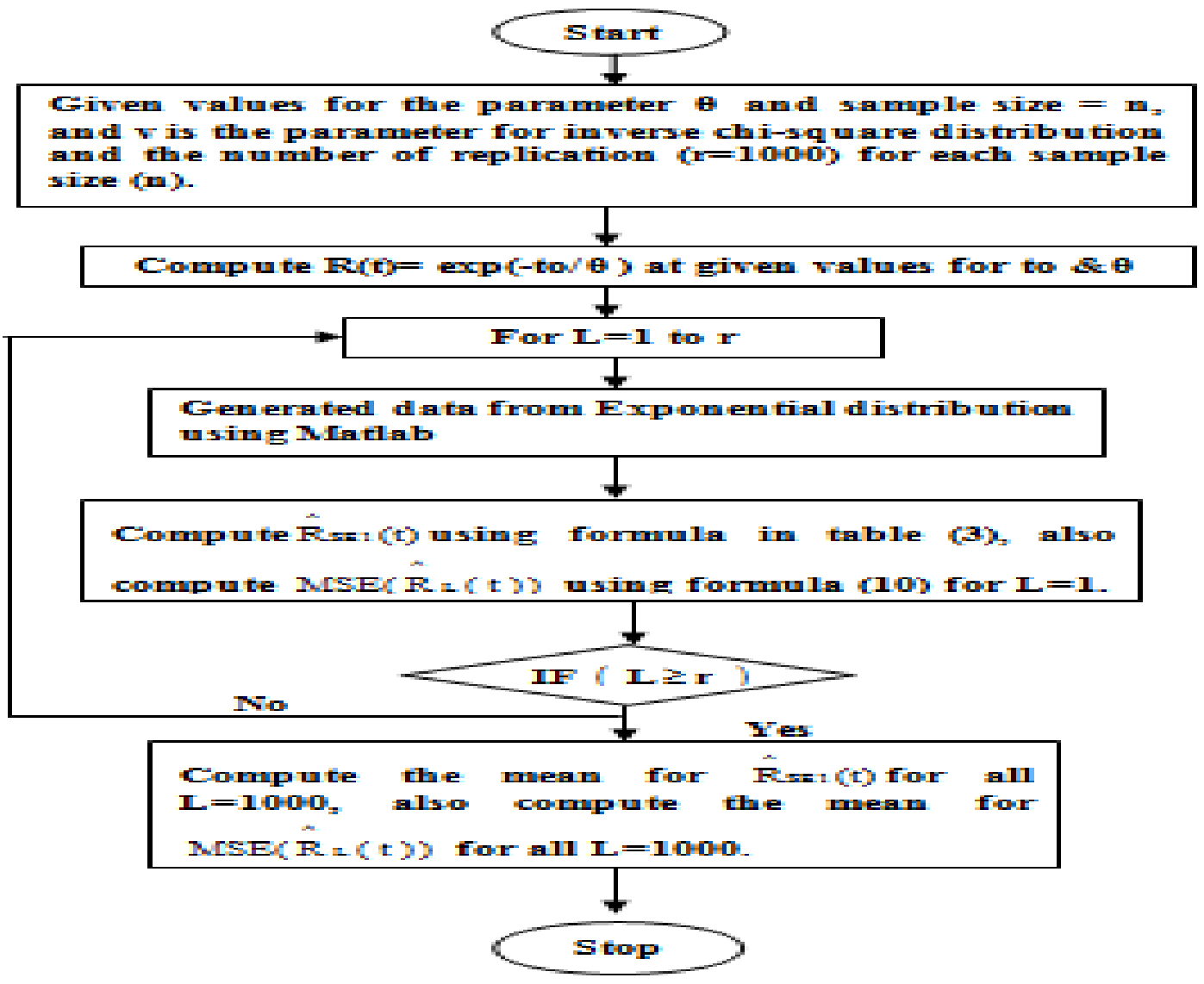

Note (1): we can reformulate the Algorithm (1) to compute Bayes estimators for $\hat{\mathrm{R}}_{\mathrm{SEi}}(\mathrm{t}), \mathrm{i}=2,3,4$ under using other distributions as prior distribution for $\theta$ with $\operatorname{MSE}$ for $\hat{\mathrm{R}}_{\mathrm{SEi}}(\mathrm{t}), \mathrm{i}=2,3,4$. 


\section{Comparison Bayes Estimators of Reliability in the Exponential Distribution}

Algorithm (2): To compute Bayes estimators ( $\mathrm{R}_{\mathrm{WSE} 1}(\mathrm{t})$ ) using inverse chisquare as prior distribution for $\theta$ with MWSE for $\mathrm{R}_{\mathrm{WSEl}}(\mathrm{t})$.

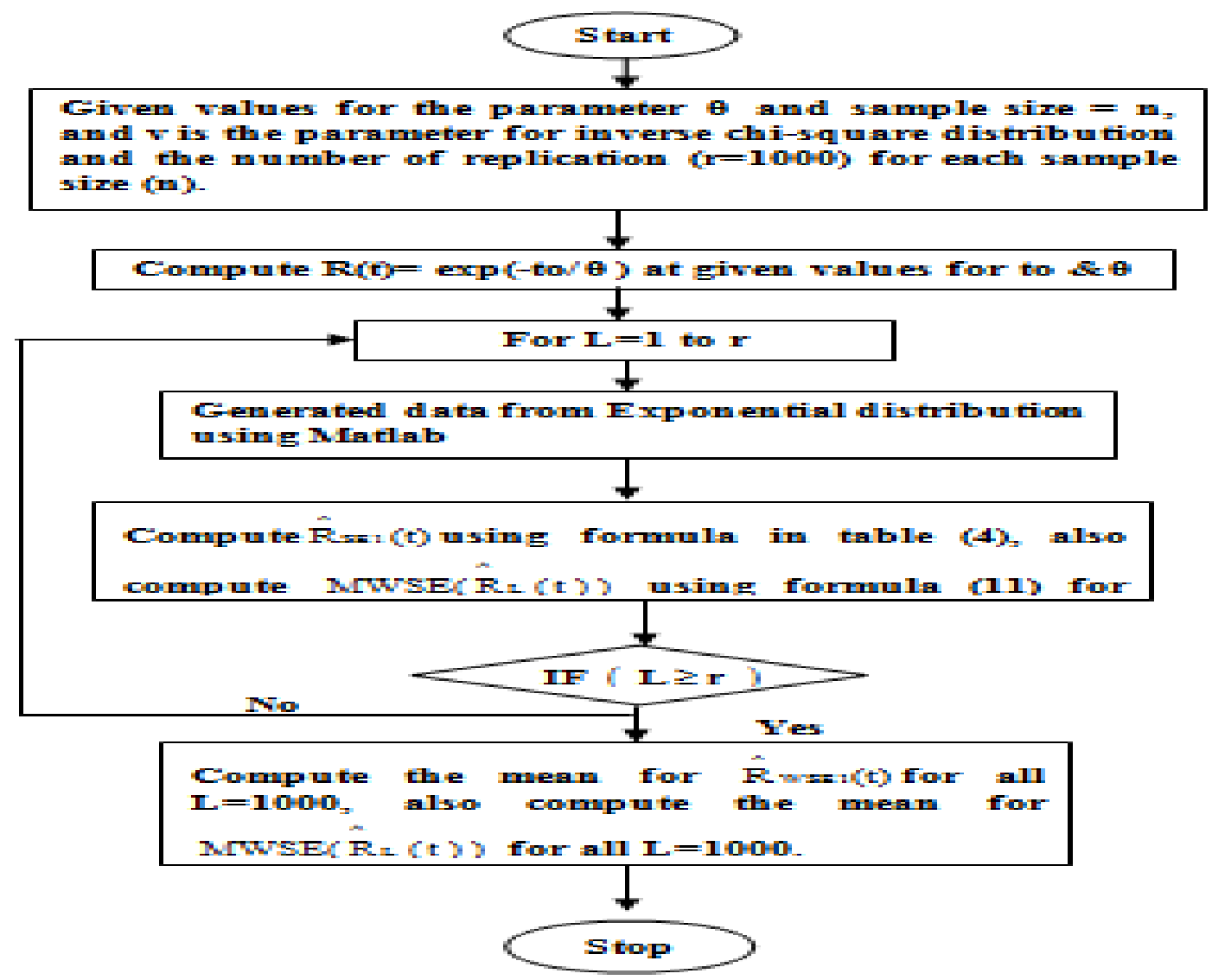

Note (2): we can reformulate the Algorithm (2) to compute Bayes estimators for $\mathrm{R}_{\text {WSEi }}(\mathrm{t}), \mathrm{i}=2,3,4$ under using other distributions as prior distribution for $\theta$ with

MWSE for $\mathrm{R}_{\text {wSEi }}(\mathrm{t}), \mathrm{i}=2,3,4$. 


\section{Comparison Bayes Estimators of Reliability in the Exponential Distribution}

Appendix-E: The summarized and tabulated discussions and conclusions.

Table 5-1: Best Estimation according to the smallest value for $\operatorname{MSE}(R(t))$.

\begin{tabular}{|c|c|c|c|c|c|c|c|c|c|c|c|c|c|}
\hline \multirow{3}{*}{\multicolumn{4}{|c|}{ parameters }} & \multirow{2}{*}{\multicolumn{4}{|c|}{$\begin{array}{c}\operatorname{MSE}(\hat{R}(\mathbf{t})) \\
\text { Sample Size(n) }\end{array}$}} & \multirow{2}{*}{\multicolumn{2}{|c|}{ Par. }} & \multirow{2}{*}{\multicolumn{4}{|c|}{$\begin{array}{c}\operatorname{MSE}(\hat{R}(\mathbf{t})) \\
\text { Sample Size(n) }\end{array}$}} \\
\hline & & & & & & & & & & & & & \\
\hline & & & & 30 & 60 & 90 & 120 & & & 30 & 60 & 90 & 120 \\
\hline \multirow[t]{4}{*}{0.5} & 0.5 & 4 & & 0.00377 & 0.00209 & 0.00135 & 0.00111 & 5 & 2 & 0.00314 & 0.00190 & 0.00127 & 0.00106 \\
\hline & 1.5 & 6 & & 0.0 & 0.00032 & 0.00021 & 0.00 & 5 & 2 & & 0.00032 & 0.00020 & \\
\hline & 2.5 & 6 & & $4.515 \mathrm{e}^{-5}$ & $2.1844 \mathrm{e}^{-5}$ & $1.2897 \mathrm{e}^{5}$ & $1.0665 e^{-5}$ & 5 & 2 & $4.8983 \mathrm{e}^{-5}$ & $2.3362 \mathrm{e}^{5}$ & $1.3592 \mathrm{e}^{-5}$ & $1.1113 \mathrm{e}^{-5}$ \\
\hline & 3.5 & 6 & & $3.708 \mathrm{e}^{-6}$ & $1.3354 \mathrm{e}^{-6}$ & $6.7785 \mathrm{e}^{-7}$ & 5.31 & 5 & 2 & $4.0753 \mathrm{e}^{-6}$ & $1.4735 \mathrm{e}^{-6}$ & $7.401 \mathrm{e}^{-7}$ & $5.7078 \mathrm{e}^{-7}$ \\
\hline \multirow{3}{*}{0.5} & 1.5 & 9 & 3 & & 0.00026 & 0.00 & 160 & 3 & & 059 & 0.1 & 021 & 18 \\
\hline & 2.5 & 9 & 3 & $2.5101 \mathrm{e}^{-6}$ & $1.5801 \mathrm{e}^{-6}$ & $1.0369 \mathrm{e}^{-6}$ & $9.0209 \mathrm{e}^{-6}$ & 3 & & $4.8655 \mathrm{e}^{-6}$ & $2.2661 \mathrm{e}^{-6}$ & $1.3208 \mathrm{e}^{-6}$ & $1.0857 \mathrm{e}^{-6}$ \\
\hline & 3.5 & 9 & 3 & $1.7018 \mathrm{e}^{-6}$ & $8.6714 \mathrm{e}^{-7}$ & $5.0366 \mathrm{e}^{-7}$ & $4.2407 e^{-7}$ & 3 & & $4.0843 e^{-6}$ & $1.3974 \mathrm{e}^{-6}$ & $6.9743 \mathrm{e}^{-7}$ & $5.4311 \mathrm{e}^{-7}$ \\
\hline$\theta$ & $\mathbf{t}$ & $\mathbf{v}$ & - & \multicolumn{4}{|c|}{ Inverse Chi-square distribution } & $\alpha$ & $\beta$ & \multicolumn{4}{|c|}{ Inverted Gamma distribution } \\
\hline \multirow[t]{4}{*}{1} & 0.5 & 4 & & 0.00315 & 0.00158 & 0.00108 & 0.0 & 3 & $\mathbf{1}$ & 0.00326 & 0.00161 & 0.00109 & 0.00081 \\
\hline & 1.5 & 4 & & & 0.00170 & 0.0 & & 3 & $\mathbf{1}$ & 0.0 & & 119 & \\
\hline & 2.5 & 6 & & 103 & 0.00060 & 0.0 & 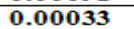 & 5 & 2 & 094 & 0.00057 & 0.00041 & 032 \\
\hline & 3.5 & 6 & & 0.00028 & 0.00016 & 0.00011 & $9.0178 \mathrm{e}^{-5}$ & 5 & 2 & 0.00024 & 0.00015 & 0.00010 & $8.6466 \mathrm{e}^{-6}$ \\
\hline $\bar{\theta}$ & $\mathbf{t}$ & $\mathbf{a}$ & $\mathbf{b}$ & \multicolumn{4}{|c|}{ Improper distribution } & $\mathbf{c}$ & - & \multicolumn{4}{|c|}{\begin{tabular}{|l|} 
Non- informative distribution \\
\end{tabular}} \\
\hline \multirow[t]{4}{*}{1} & 0.5 & 7 & 3 & 396 & 0.00183 & 0.00120 & 0.00087 & $\mathbf{1}$ & & 0.00284 & 0150 & 0103 & 0.00077 \\
\hline & 1.5 & 7 & 3 & 324 & 0.00175 & 0.00123 & & 3 & & 0.00319 & 174 & 0.00121 & 0.00092 \\
\hline & 2.5 & 7 & 3 & 0.00093 & 0.00056 & 0.00041 & 0.000320 & 3 & & 0.00109 & 0.00062 & 0.00043 & 0.00034 \\
\hline & 3.5 & 7 & 3 & 0.00021 & 0.00014 & 0.00010 & $8.3143 e^{-5}$ & 3 & & 0.00031 & 0.00017 & 0.00011 & $9.296 \mathrm{e}^{-5}$ \\
\hline
\end{tabular}

\section{Continue for Table 5-1}

\begin{tabular}{|c|c|c|c|c|c|c|c|c|c|c|c|c|c|}
\hline \multirow{2}{*}{\multicolumn{4}{|c|}{ parameters }} & \multirow{2}{*}{\multicolumn{4}{|c|}{$\begin{array}{c}\operatorname{MSE}(\hat{R}(\mathbf{t})) \\
\text { Sample Size(n) }\end{array}$}} & \multirow{3}{*}{\multicolumn{2}{|c|}{ Par. }} & \multicolumn{4}{|c|}{$\operatorname{MSE}(\hat{R}(t))$} \\
\hline & & & & & & & & & & \multicolumn{4}{|c|}{ Sample Size(n) } \\
\hline & & & & 30 & 60 & 90 & 120 & & & 30 & 60 & 90 & 120 \\
\hline$\theta$ & $\mathbf{t}$ & $\mathbf{v}$ & - & \multicolumn{4}{|c|}{ Inverse Chi-square distribution } & $\alpha$ & $\beta$ & \multicolumn{4}{|c|}{ Inverted Gamma distribution } \\
\hline \multirow[t]{4}{*}{1.5} & 0.5 & 4 & & 0.00245 & 0.00111 & 0.00069 & 0.00051 & 3 & $\mathbf{I}$ & 0.00265 & 0.00117 & 0.00071 & 0.00052 \\
\hline & 1.5 & 4 & & 0.00460 & 0.00232 & 0.00151 & 0.00114 & 3 & 1 & 0.00481 & 0.00239 & 0.00154 & 0.00115 \\
\hline & 2.5 & 4 & & 0.00292 & 0.00157 & 0.00105 & 0.00080 & 3 & $\mathbf{1}$ & 0.00292 & 0.00158 & 0.00105 & 0.00080 \\
\hline & 3.5 & 6 & & 0.00138 & 0.00078 & 0.00052 & 0.00040 & 5 & 2 & 0.00134 & 0.00077 & 0.00052 & 0.00040 \\
\hline$\theta$ & $\mathbf{t}$ & $\mathbf{a}$ & $\mathbf{b}$ & \multicolumn{4}{|c|}{ Improper distribution } & c & - & \multicolumn{4}{|c|}{ Non-informative distribution } \\
\hline \multirow[t]{4}{*}{1.5} & 0.5 & 7 & 3 & 0.00378 & 0.00151 & 0.00087 & 0.00061 & $\mathbf{I}$ & & 0.00207 & 0.00100 & 0.00065 & 0.00048 \\
\hline & 1.5 & 7 & 3 & 0.00638 & 0.00291 & 0.00177 & 0.00129 & $\mathbf{1}$ & & 0.00438 & 0.00223 & 0.00149 & 0.00112 \\
\hline & 2.5 & 7 & 3 & 0.00351 & 0.00179 & 0.00114 & 0.00086 & 3 & & 0.00300 & 0.00160 & 0.00106 & 0.00081 \\
\hline & 3.5 & 7 & 3 & 0.00143 & 0.00080 & 0.00053 & 0.00041 & 3 & & 0.00143 & 0.00079 & 0.00053 & 0.00041 \\
\hline$\theta$ & $\mathbf{t}$ & $\mathbf{v}$ & - & \multicolumn{4}{|c|}{ Inverse Chi-square distribution } & $\alpha$ & $\beta$ & \multicolumn{4}{|c|}{ Inverted Gamma distribution } \\
\hline \multirow[t]{4}{*}{2.5} & 0.5 & 4 & & 0.00119 & 0.00057 & 0.00033 & 0.00026 & 3 & 1 & 0.00135 & 0.00061 & 0.00035 & 0.00027 \\
\hline & 1.5 & 4 & & 0.00408 & 0.00212 & 0.00128 & 0.00101 & 3 & $\mathbf{I}$ & 0.00455 & 0.00225 & 0.00133 & 0.00105 \\
\hline & 2.5 & $\mathbf{4}$ & & 0.00446 & 0.00246 & 0.00152 & 0.00121 & 3 & $\mathbf{1}$ & 0.00484 & 0.00257 & 0.00157 & 0.00124 \\
\hline & 3.5 & 4 & & 0.00354 & 0.00205 & 0.00129 & 0.00104 & 3 & 1 & 0.00374 & 0.00210 & 0.00131 & 0.00105 \\
\hline $\bar{\theta}$ & $\mathbf{t}$ & $\mathbf{a}$ & $\mathbf{b}$ & \multicolumn{4}{|c|}{ Improper distribution } & c & - & \multicolumn{4}{|c|}{ Non-informative distribution } \\
\hline \multirow[t]{4}{*}{2.5} & 0.5 & 7 & 3 & 0.00229 & 0.00087 & 0.00046 & 0.00034 & $\mathbf{1}$ & & 0.00093 & 0.00050 & 0.00031 & 0.00024 \\
\hline & 1.5 & 7 & 3 & 0.00737 & 0.00307 & 0.00170 & 0.00127 & $\mathbf{1}$ & & 0.00343 & 0.00 & 0.00121 & 0.00096 \\
\hline & 2.5 & 7 & 3 & 0.00748 & 0.00336 & 0.00193 & 0.00147 & I & & 0.00406 & 0.00235 & 0.00149 & 0.00118 \\
\hline & 3.5 & 7 & 3 & 0.00546 & 0.00262 & 0.00155 & 0.00121 & 1 & & 0.00352 & 0.00204 & 0.00130 & 0.00103 \\
\hline
\end{tabular}

Table 5-2: Best Estimation according to the smallest value for $\operatorname{MWSE}(\mathbf{R}(t))$.

\begin{tabular}{|c|c|c|c|c|c|c|c|c|c|c|c|c|c|}
\hline \multirow{2}{*}{\multicolumn{4}{|c|}{ parameters }} & \multirow{2}{*}{\multicolumn{4}{|c|}{$\begin{array}{l}\text { MNWSE(R(t)) } \\
\text { Sample Size(n) }\end{array}$}} & \multirow{3}{*}{\multicolumn{2}{|c|}{ Par. }} & \multicolumn{4}{|c|}{ MWVE $\hat{R}(t))$} \\
\hline & & & & & & & & & & \multicolumn{4}{|c|}{ Sample Size(n) } \\
\hline & & & & 30 & 60 & 90 & 120 & & & 30 & 60 & 90 & 120 \\
\hline$\theta$ & $\mathbf{t}$ & $\mathbf{v}$ & - & \multicolumn{4}{|c|}{ Inverse Chi-square distribution } & $\alpha$ & $\bar{\beta}$ & \multicolumn{4}{|c|}{ Inverted Gamma distribution } \\
\hline \multirow[t]{4}{*}{0.5} & 0.5 & 4 & & 0.01183 & 0.00611 & 0.00389 & 0.00315 & 5 & 2 & 0.00978 & 0.00554 & 0.00364 & 0.00300 \\
\hline & 1.5 & 4 & & 0.01177 & 0.00665 & 0.00437 & 0.00363 & 5 & 2 & 0.01008 & 0.00609 & 0.00411 & 0.00347 \\
\hline & 2.5 & 6 & & 0.00365 & 0.00223 & 0.00152 & 0.00130 & 5 & 2 & 0.00338 & 0.00215 & 0.00146 & 0.00127 \\
\hline & 3.5 & 6 & & 0.00086 & 0.00054 & 0.00037 & 0.00033 & 5 & 2 & 0.00086 & 0.00055 & 0.00037 & 0.00034 \\
\hline $\bar{\theta}$ & $\mathbf{t}$ & $\mathbf{a}$ & $\mathbf{b}$ & \multicolumn{4}{|c|}{ Improper distribution } & $\mathbf{c}$ & - & \multicolumn{4}{|c|}{ Non-informative distribution } \\
\hline \multirow[t]{4}{*}{0.5} & 0.5 & 7 & 3 & 0.00870 & 0.00520 & 0.00349 & 0.00290 & $\mathbf{1}$ & & 0.01170 & 0.00607 & 0.00385 & 0.00313 \\
\hline & 1.5 & 7 & 3 & 0.00914 & 0.00575 & 0.00395 & 0.00336 & 3 & & 0.01262 & 0.00689 & 0.00452 & 0.00371 \\
\hline & 2.5 & 9 & 3 & 0.00297 & 0.00194 & 0.00138 & 0.00120 & 3 & & 0.00382 & 0.00229 & 0.00155 & 0.00132 \\
\hline & 3.5 & 9 & 3 & 0.00063 & 0.00045 & 0.00033 & 0.00030 & 3 & & 0.00090 & 0.00056 & 0.00038 & 0.00034 \\
\hline$\theta$ & $\mathbf{t}$ & $\mathbf{v}$ & - & \multicolumn{4}{|c|}{ Inverse Chi-square distribution } & $\alpha$ & $\beta$ & \multicolumn{4}{|c|}{ Inverted Gamma distribution } \\
\hline \multirow[t]{4}{*}{$\mathbf{I}$} & 0.5 & 4 & & 0.00578 & 0.00276 & 0.00185 & 0.00136 & 3 & $\mathbf{I}$ & 0.00401 & 0.00171 & 0.00103 & 0.00075 \\
\hline & 1.5 & 4 & & 0.01639 & 0.00838 & 0.00574 & 0.00432 & 3 & $\mathbf{I}$ & 0.01582 & 0.00727 & 0.00452 & 0.00334 \\
\hline & 2.5 & 4 & & 0.01469 & 0.00797 & 0.00554 & 0.00426 & 3 & $\mathbf{1}$ & 0.01955 & 0.00962 & 0.00612 & 0.00459 \\
\hline & 3.5 & 4 & & 0.00952 & 0.00542 & 0.00381 & 0.00299 & 3 & 1 & 0.01730 & 0.00906 & 0.00586 & 0.00448 \\
\hline$\theta$ & $\mathbf{t}$ & $\mathbf{a}$ & $\mathbf{b}$ & \multicolumn{4}{|l|}{ Improper } & $\mathbf{c}$ & - & \multicolumn{4}{|c|}{ Non-informative distribution } \\
\hline $\mathbf{1}$ & 0.5 & $\frac{4}{7}$ & 3 & 0.00738 & 0.00325 & 0.00210 & 0.00151 & 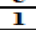 & & 0.00502 & 0.00256 & 0.00175 & 0.00130 \\
\hline & 1.5 & 7 & 3 & 0.01942 & 0.00938 & 0.00625 & 0.00464 & 1 & & 0.01545 & 0.00814 & 0.00560 & 0.00424 \\
\hline & 2.5 & 7 & 3 & 0.01609 & 0.00843 & 0.00579 & 0.00442 & 1 & & 0.01504 & 0.00810 & 0.00558 & 0.00428 \\
\hline & 3.5 & 7 & 3 & 0.00959 & 0.00541 & 0.00382 & 0.00299 & 3 & & 0.00978 & 0.00549 & 0.00385 & 0.00301 \\
\hline
\end{tabular}




\section{Comparison Bayes Estimators of Reliability in the Exponential Distribution}

Continue for Table 5-2

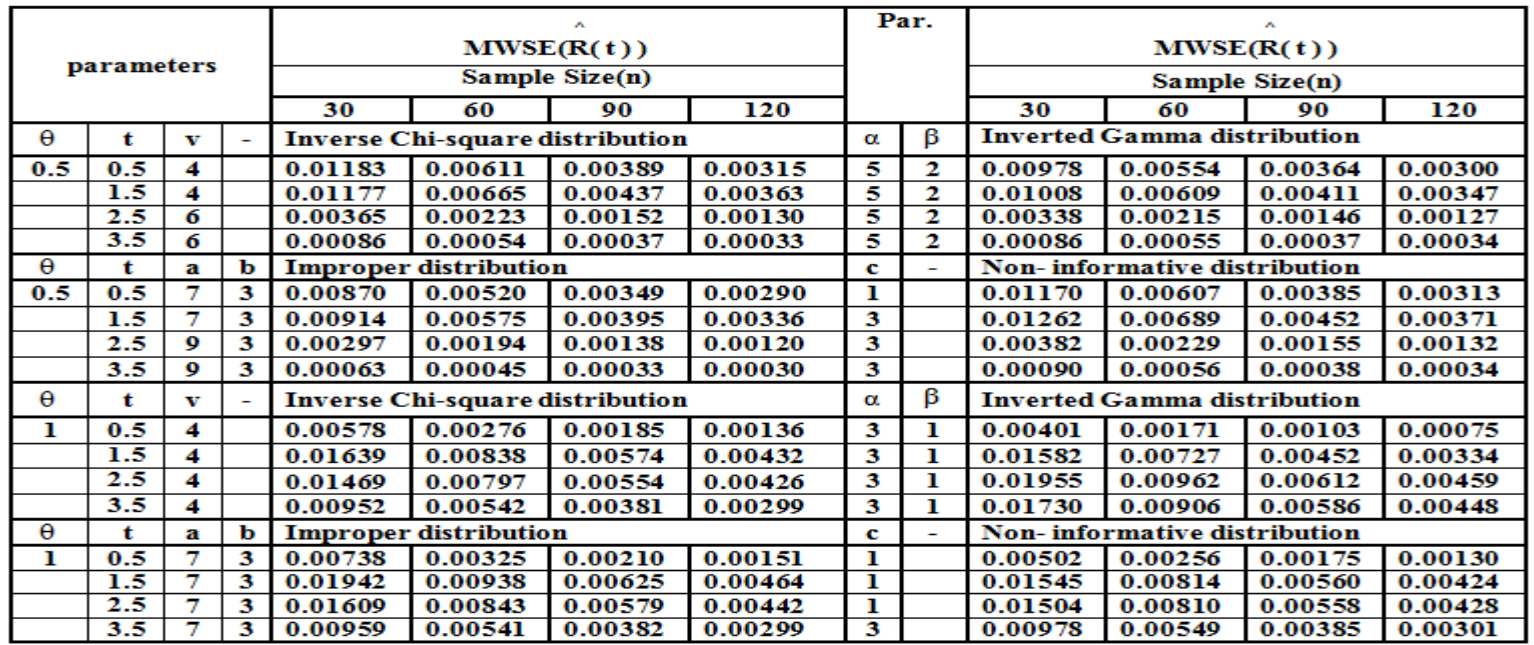

Table 6-1: the summary of conclusions for $\operatorname{MSE}(\mathbf{R}(t))$.

\begin{tabular}{|c|c|c|c|c|c|c|}
\hline \multirow[t]{3}{*}{$\theta$} & \multirow[t]{3}{*}{$t$} & \multirow{3}{*}{$\begin{array}{l}\text { The best estimation according to the smallest } \\
\text { value for } \operatorname{MSE}(\hat{R}(t)) \text { when the Prior } \\
\text { distribution is }\end{array}$} & \multirow{2}{*}{\multicolumn{4}{|c|}{$\begin{array}{c}\operatorname{MSE}(\hat{R}(\mathbf{t})) \\
\text { Sample Size(n) }\end{array}$}} \\
\hline & & & & & & \\
\hline & & & 30 & 60 & 90 & 120 \\
\hline \multirow[t]{4}{*}{0.5} & 0.5 & Improper distribution with $(a=7, b=3)$ & 0.00280 & 0.00178 & 0.00121 & .00102 \\
\hline & 1.5 & Improper distribution with $(a=9, b=3)$ & 0.00039 & 0.00026 & 0.00018 & 16 \\
\hline & 2.5 & Improper distribution with $(a=9, b=3)$ & & & & \\
\hline & 3.5 & Improper distribution with $(a=9, b=3)$ & $8 \mathrm{e}^{-6}$ & $14 e^{-7}$ & $5.0366 \mathrm{e}^{-7}$ & $7 e^{-7}$ \\
\hline$\theta$ & $\mathbf{t}$ & & & & & \\
\hline \multirow[t]{4}{*}{$\mathbf{I}$} & 0.5 & Non- informative distribution with $(c=1)$ & 0.00284 & 0.00150 & 0.00103 & 0.00077 \\
\hline & 1.5 & Inverted Gamma distribution with $(\alpha=3, \beta=1)$ & 0.00300 & 0.00168 & 0.00119 & 0.00091 \\
\hline & 2.5 & Improper distribution with $(a=7, b=3)$ & & 0.00056 & & 032 \\
\hline & 3.5 & Improper distribution with $(a=7, b=3)$ & 21 & 0.00014 & 0.00010 & $3 \mathrm{e}^{-6}$ \\
\hline$\theta$ & $\mathbf{t}$ & & & & & \\
\hline \multirow[t]{4}{*}{1.5} & 0.5 & Non-informative distribution & 207 & 0.00100 & 0.00065 & 0.00048 \\
\hline & 1.5 & Non- informative distribution with $(c=1)$ & 0.00438 & 0.00223 & 0.00149 & 0.00112 \\
\hline & 2.5 & Inverse Chi-square distribution with $(v=4)$ & 0.00292 & 0.00157 & 105 & 0.00080 \\
\hline & 3.5 & Inverted Gamma distribution with $(\alpha=5, \beta=2)$ & 0.00134 & 0.00077 & 0.00052 & 0.00040 \\
\hline$\theta$ & $\mathbf{t}$ & & & & & \\
\hline \multirow[t]{4}{*}{2.5} & 0.5 & distribution & 93 & 050 & 031 & 24 \\
\hline & 1.5 & Non- informative distribution with $(\mathrm{c}=1)$ & 0.00343 & 0.00194 & 0.00121 & 0.00096 \\
\hline & 2.5 & Non- informative distribution with $(c=1)$ & 406 & 0.00235 & 0.00149 & 0.00118 \\
\hline & 3.5 & Non- informative distribution with $(c=1)$ & 0.00352 & 0.00204 & 0.00130 & 0.00103 \\
\hline
\end{tabular}

Table 6-2: the summary of conclusions for $\operatorname{MWSE}(R(t))$.

\begin{tabular}{|c|c|c|c|c|c|c|}
\hline \multirow[t]{2}{*}{$\theta$} & \multirow[t]{2}{*}{$\mathbf{t}$} & \multirow{2}{*}{$\begin{array}{l}\text { The best estimation according to the smallest } \\
\text { value for MWSE(R(t)) when the Prior } \\
\text { distribution is }\end{array}$} & \multirow{2}{*}{\multicolumn{4}{|c|}{$\begin{array}{l}\text { MUVSE(R(t)) } \\
\text { Sample Size(n) }\end{array}$}} \\
\hline & & & & & & \\
\hline \multirow[t]{4}{*}{0.5} & 0.5 & Improper distribution with $(\mathbf{a}=7, \mathbf{b}=\mathbf{3})$ & 0.00870 & 0.00520 & 0.00349 & 0.00290 \\
\hline & 1.5 & Improper distribution with $(a=7, b=3)$ & 0.00914 & 0.00575 & 0.00395 & 0.00336 \\
\hline & 2.5 & Improper distribution with $(a=9, b=3)$ & 0.00297 & 0.00194 & 138 & o. \\
\hline & 3.5 & Improper distribution with $(a=9, b=3)$ & 0.00063 & 0.00045 & 0.00033 & 0.00030 \\
\hline $\bar{\theta}$ & 7 & & & & & \\
\hline \multirow[t]{4}{*}{ 1 } & 0.5 & Inverted Gamma distribution with $(\alpha=3, \beta=1)$ & 0.00401 & 0.00171 & 0.00103 & 0.00075 \\
\hline & 1.5 & Inverted Gamma distribution with $(\alpha=3, \beta=1)$ & 0.01582 & 0.00727 & 0.00452 & 0.00334 \\
\hline & 2.5 & Inverse Chi-square distribution with $(v=4)$ & 0.01469 & 0.00797 & 0.00554 & 0.00426 \\
\hline & 3.5 & Inverse Chi-square distribution with $(v=4)$ & 0.00952 & 0.00542 & 0.00381 & 0.00299 \\
\hline$\theta$ & $\mathbf{t}$ & & & & & \\
\hline \multirow[t]{4}{*}{1.5} & 0.5 & Non- informative distribution with $(c=1)$ & 0.00305 & 0.00144 & 0.00092 & 0.00068 \\
\hline & 1.5 & Non-informative distribution with $(c=1)$ & 306 & o.0 & 417 & 313 \\
\hline & 2.5 & Non- informative distribution with $(c=1)$ & 0.01752 & 0.00889 & o. & 442 \\
\hline & 3.5 & Non- informative distribution with $(\mathrm{c}=1)$ & 0.01682 & 0.00877 & 0.00577 & 0.00441 \\
\hline $\bar{\theta}$ & $\mathbf{t}$ & & & & & \\
\hline \multirow[t]{4}{*}{2.5} & 0.5 & ative distribution with $(c=1)$ & 118 & 0.00063 & 0.00038 & 0.00030 \\
\hline & 1.5 & Non- informative distribution with $(c=1)$ & & & 0.00227 & \\
\hline & 2.5 & Non-informative distribution & & & & \\
\hline & 3.5 & Non-informative distribution with $(c=1)$ & 0.0156 & 0.00867 & 0.00541 & 0.00430 \\
\hline
\end{tabular}




\section{Comparison Bayes Estimators of Reliability in the Exponential Distribution}

\section{مقارنت مقدرات بيز للمعوليتّ في التوزيع الاسي}

$$
\text { أ.م.د. جنان عباس ناصر العبيدي /الكليت التقنيت الاداريتّ / بغداد }
$$

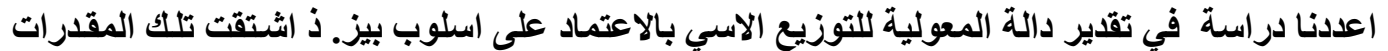

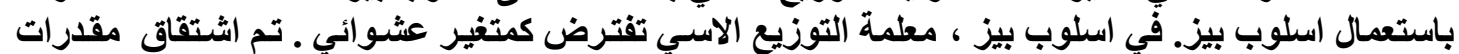

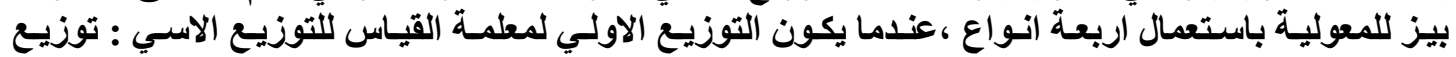

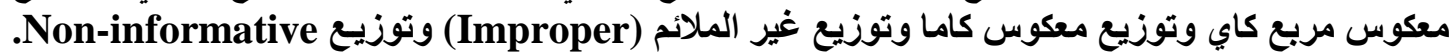

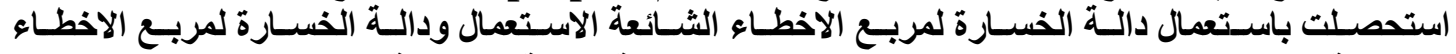

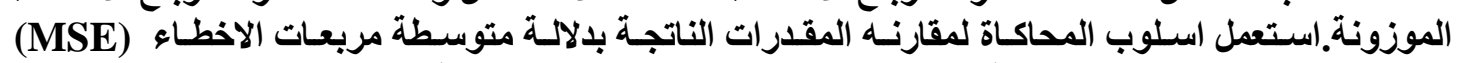

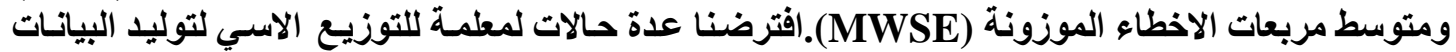

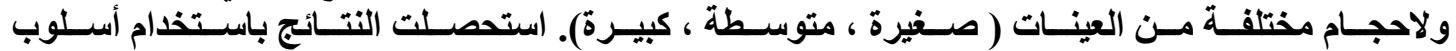

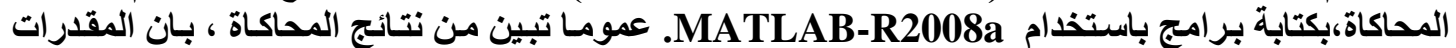

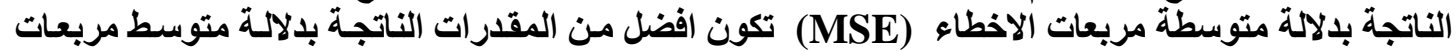

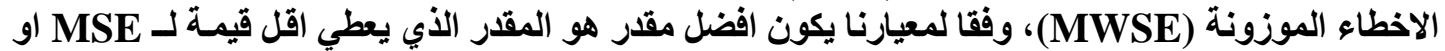

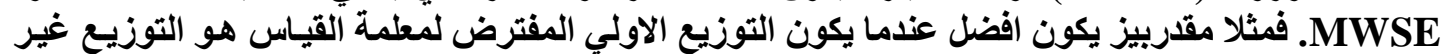

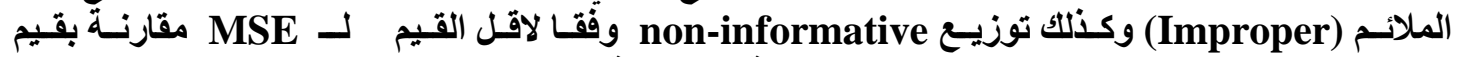

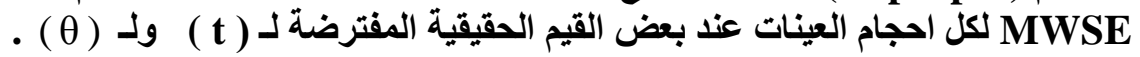

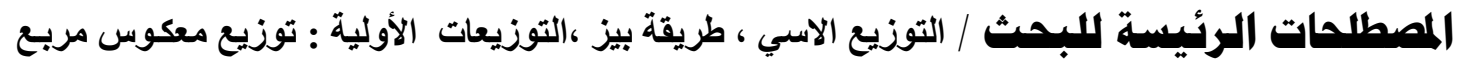

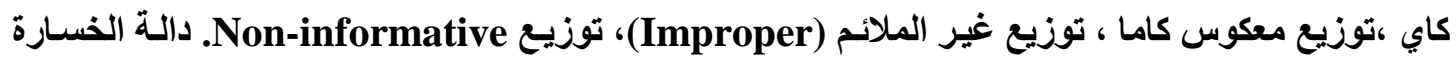

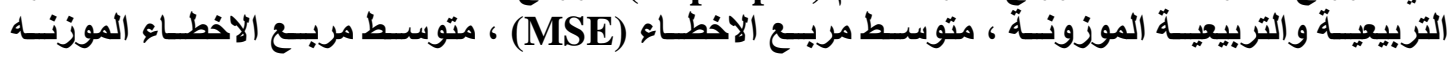
(MWSE) 